\title{
Heavy, Flavour Production, QCD, and the Quark-Photon Coupling
}

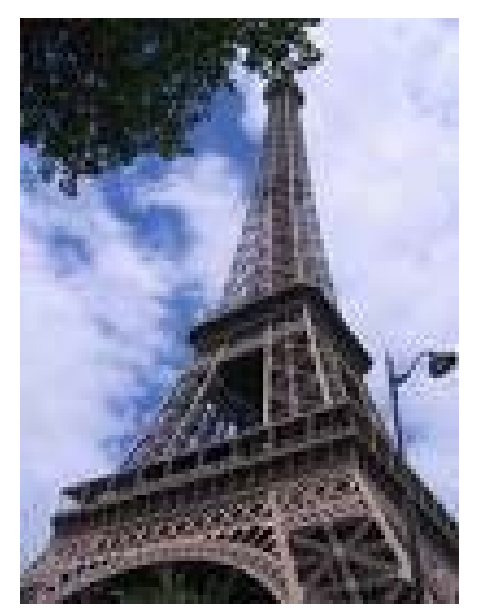

Achim Geiser, DESY Hamburg

Achim.Geiser@desy.de

Photon2007

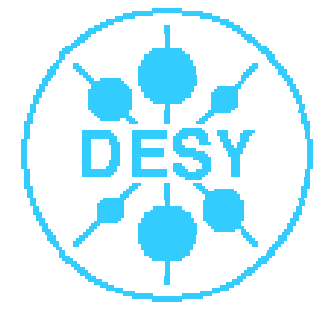

Paris, France, 11 July 2007

Introduction: Heavy Quark photoproduction

- Choice of renormalization/factorization scales in QCD:

Phenomenological considerations and cross checks with data Plea for a change of default QCD scale for NLO calculations

- Speculation on photon-quark coupling

- Conclusions 


\section{Photoproduction of Open Heavy Flavour}

Dominant production process in ep-collisions: Boson-Gluon -Fusion

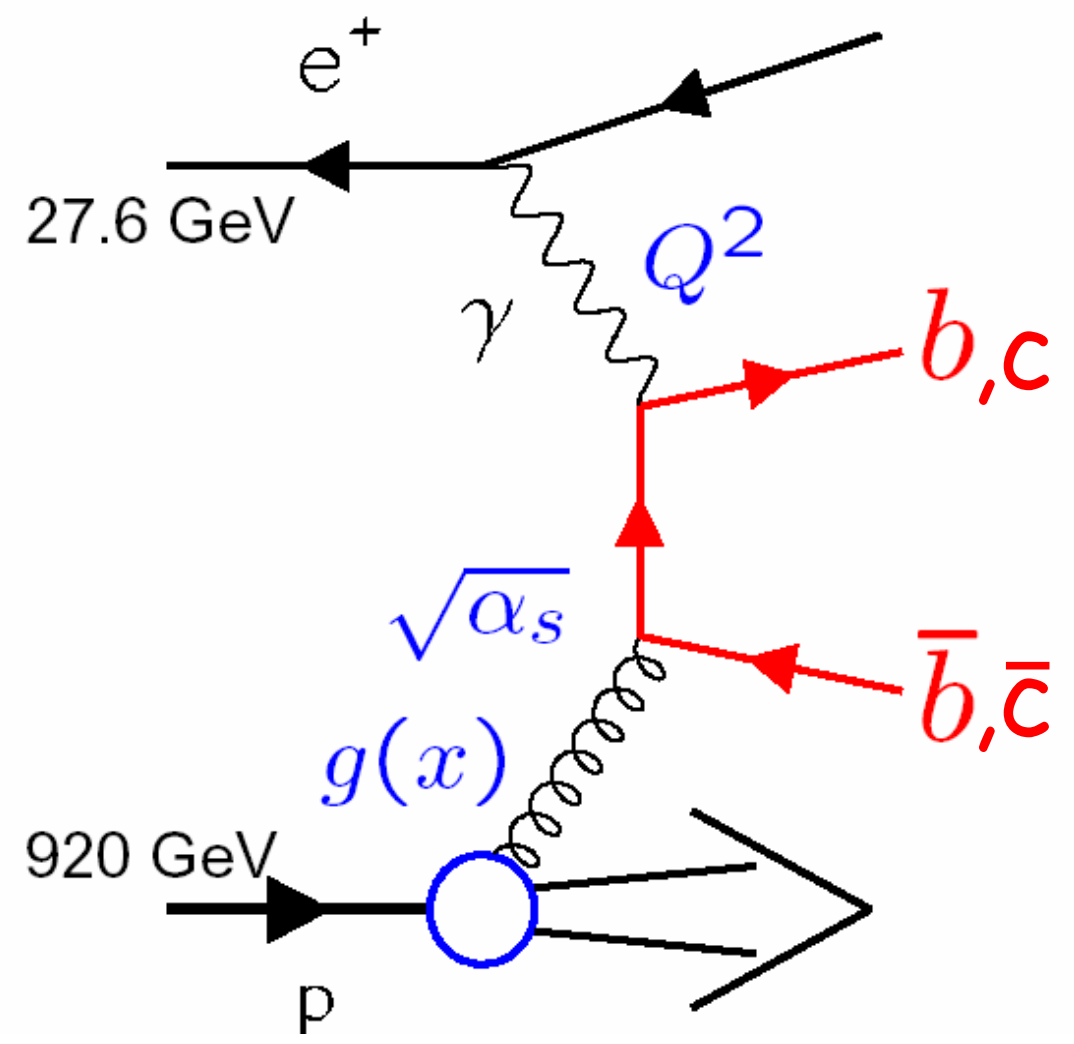

- Driven by gluons in the proton

- Relevant scales:

$$
\begin{aligned}
m_{b} & \sim 5 \mathrm{GeV}^{2} m_{c} \sim 1.5 \mathrm{GeV} \\
Q^{2} & \lesssim 1 \mathrm{GeV}^{2} \rightarrow \gamma p \\
& >2 \mathrm{GeV}^{2} \rightarrow \text { DIS } \\
p_{T^{,}, c} & \text { Event selection: } p_{t}^{\text {jet }}>6 \text { or } 7 \mathrm{GeV}
\end{aligned}
$$

multiscale problem

-> terms $\left[\alpha_{\mathrm{s}} \ln \left(\mathrm{Q}^{2} / \mathrm{m}_{\mathrm{Q}}{ }^{2}\right)\right]^{\mathrm{n}}, \quad\left[\alpha_{\mathrm{s}} \ln \left(\mathrm{p}_{\mathrm{T}}{ }^{2} / \mathrm{m}_{\mathrm{Q}}{ }^{2}\right)\right]^{\mathrm{n}}$, etc. in perturbative expansion $->$ potentially large th. errors 


\section{Beauty in photoproduction: summary}

details->talk J. Loizides

bquark HERA

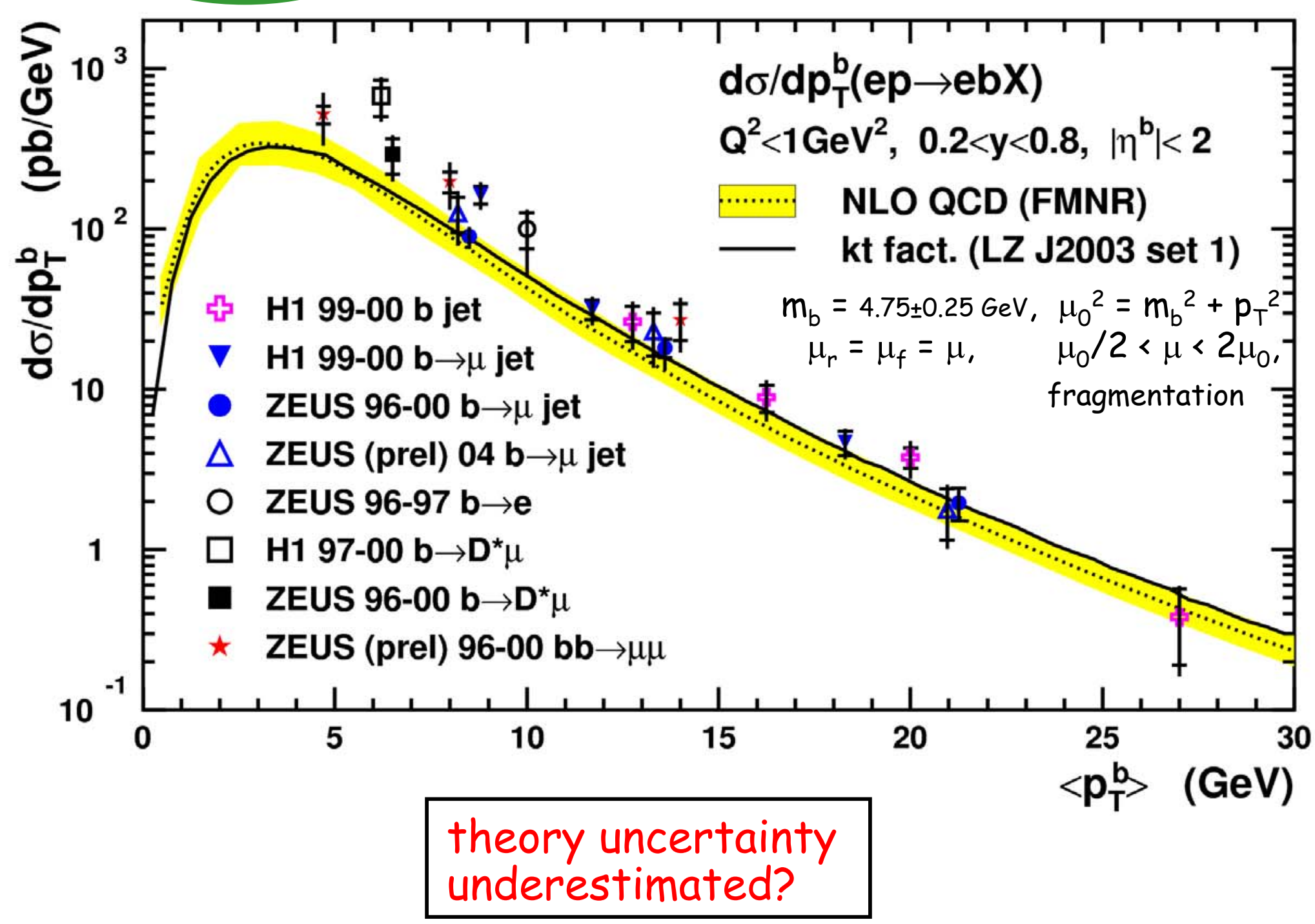

data/QCD:

reasonable agreement, but tendency data > QCD at low $P_{T}$ 
scale choice often dominant theoretical error

\section{How well do we understand choice of QCD scales?}

(issue also raised e.g. in talk G. Grindhammer) 


\section{remarks on QCD scale dependence}

Ideally (calculation to all orders) QCD predictions should not depend on the choice of renormalization and factorization scales $\mu_{r}, \mu_{f}$

$\Rightarrow$ not physical parameters $\quad \Rightarrow$ can not be determined from data

- In practice, finite order calculations do depend on choice of these scales

= reference points for perturbative expansion (Taylor expansion)

- Choice of scale is to large extent arbitrary.

Best solution is case by case evaluation of sensible scales, and detailed study of behaviour of cross section with respect to variation of these scales.

In practice often replaced by simple recipes. Overinterpretation might lead to premature conclusions that data/QCD predictions do not agree.

If recipes at all, at least try to use the "best" $\Rightarrow$ try to evaluate performance 


\section{Common recipes for scale choice}

Common sense criterion/try to minimize occurrence of large logs:

$\Rightarrow 1$. choose "natural" scale of process involved $\left(m, Q^{2}, E_{T}, \ldots\right)$ but subscales (e.g. subdominant gluon radiation) often lower

nowadays often only criterion used

Two other textbook criteria from the late 80ies: time for a revival?

principle of fastest apparent convergence: choice of scales such that, ideally, cross sections will not change when higher order corrections are included $\Rightarrow 2$. best bet: $N L O=L O \Rightarrow$ hope: NNLO $=$ NLO check!

principle of minimal sensitivity: minimize sensitivity to scale variations $\Rightarrow 3$. best bet: $d \sigma / d \mu=0 \Rightarrow$ hope: minimize NLO corrections

range of variation of scale is supposed to be a measure of theoretical error for uncalculated higher orders

evaluate all three criteria to determine a "reasonable" choice 


\section{example: total b cross section at HERA-B}

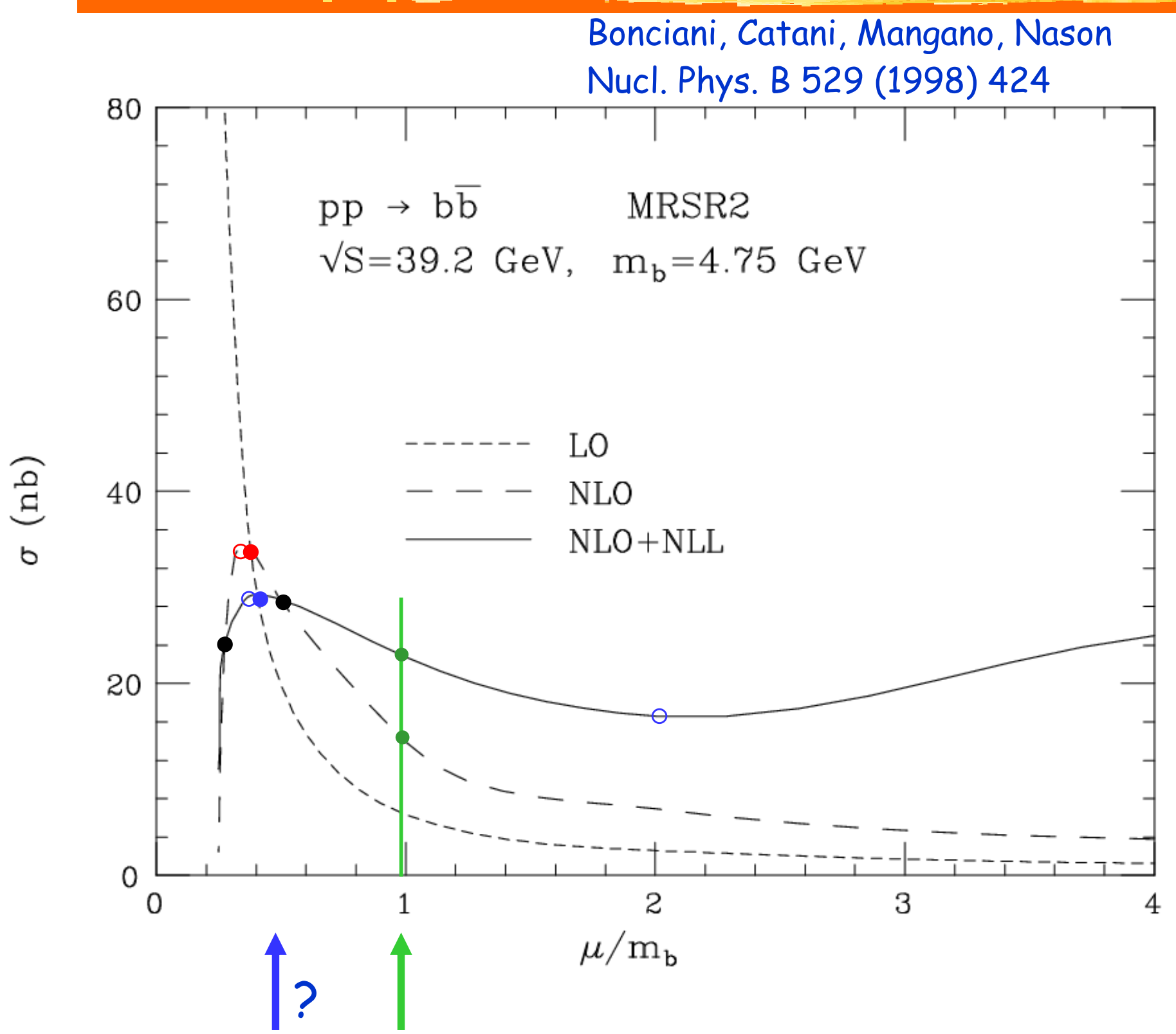

NLO stability:

- $\quad N L O=L O$

- $d \sigma_{N L O} / d \mu=0$

NLO+NLL stability:

- $N L O+N L L=L O$

- $\quad N L O+N L L=N L O$

- $d \sigma_{N L O+N L L} / d \mu=0$

_ natural" scale

in many cases, such solutions do not exist

$\Rightarrow$ consider those cases where they do 


\section{example: Higgs production at $L H C$}

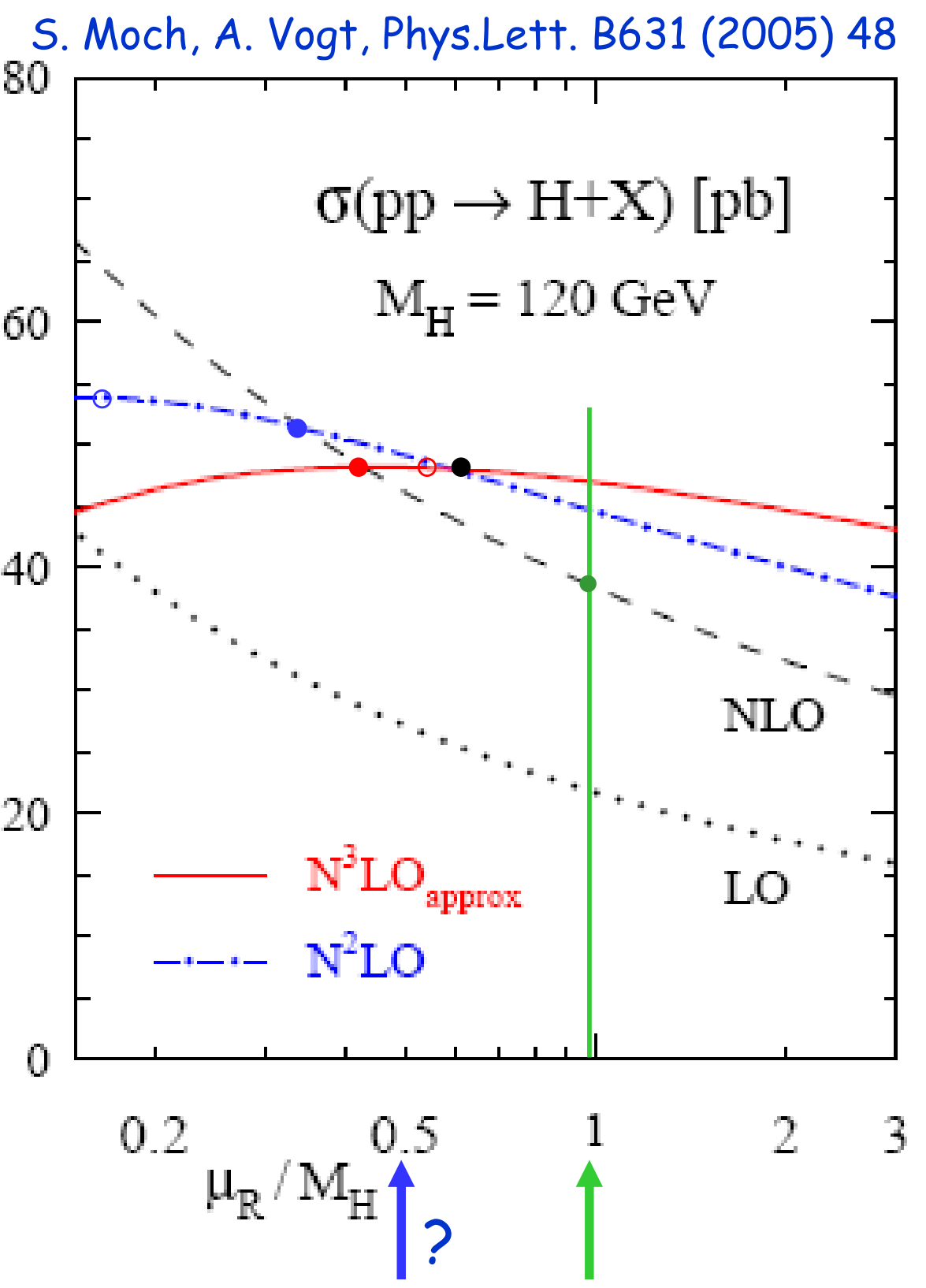

NNLO stability:

- $\quad$ NNLO = NLO

- $d \sigma_{N N L O} / d \mu=0$

NLO stability:

- $\quad N^{3} L O=N L O$

- $N^{3} L O=N N L O$

- $d \sigma_{N L O+N L L} / d \mu=0$

_ natural" scale

NNLO/N3LO calculations, where available, support validity of scheme! 


\section{"optimal" ren./fact. scale from theory}

$\mu_{0} / 2<\mu<2 \mu_{0}$ $\mu_{0} / 4<\mu<\mu_{0}$
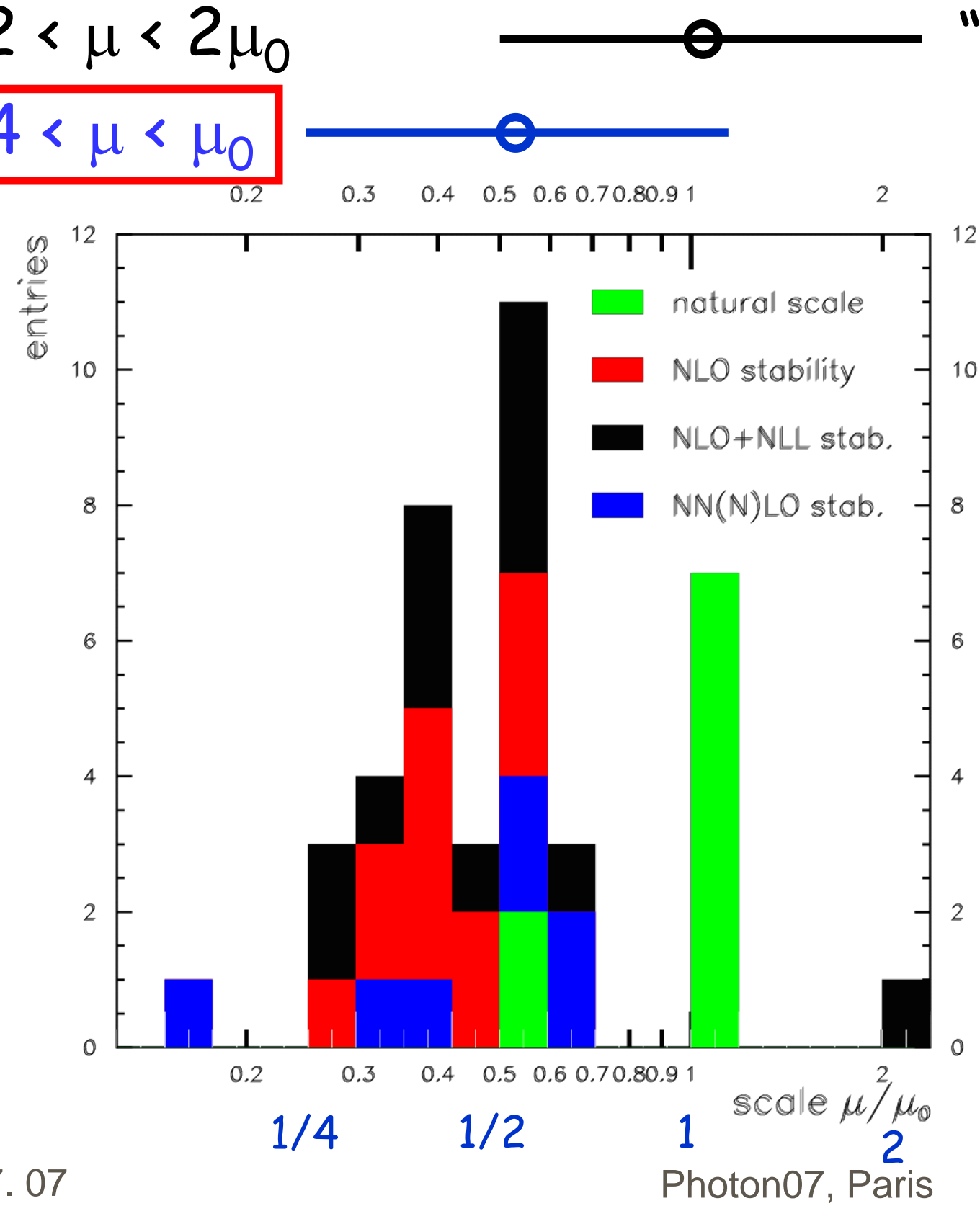

"standard" scale range proposed new default
NLO (NNLO) QCD survey of:

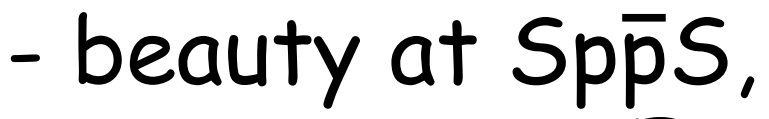
Tevatron, HERA-B

- top at Tevatron

- $\mathrm{Z}, \mathrm{H}$ at $\mathrm{LHC}$

$$
\mu_{0}^{2}=m^{2}\left(+p_{T}^{2}\right)
$$

- jets in $\gamma p$ and at Tevatron

$$
\mu_{0}{ }^{2}=E_{T^{2}}
$$




\section{cross check with data: $c$ and $b$ at Tevatron}

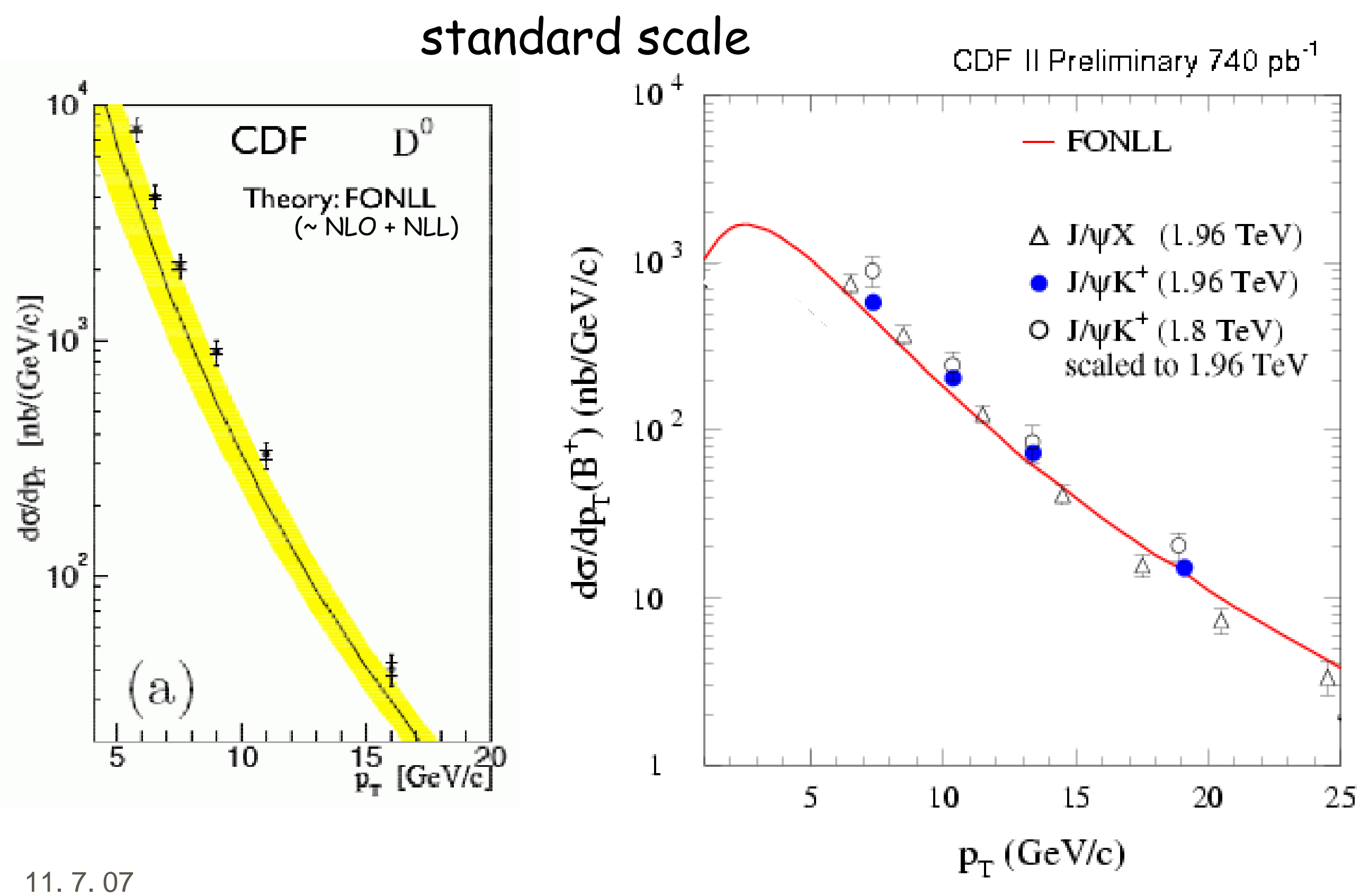




\section{cross check with data: $c$ and $b$ at Tevatron}

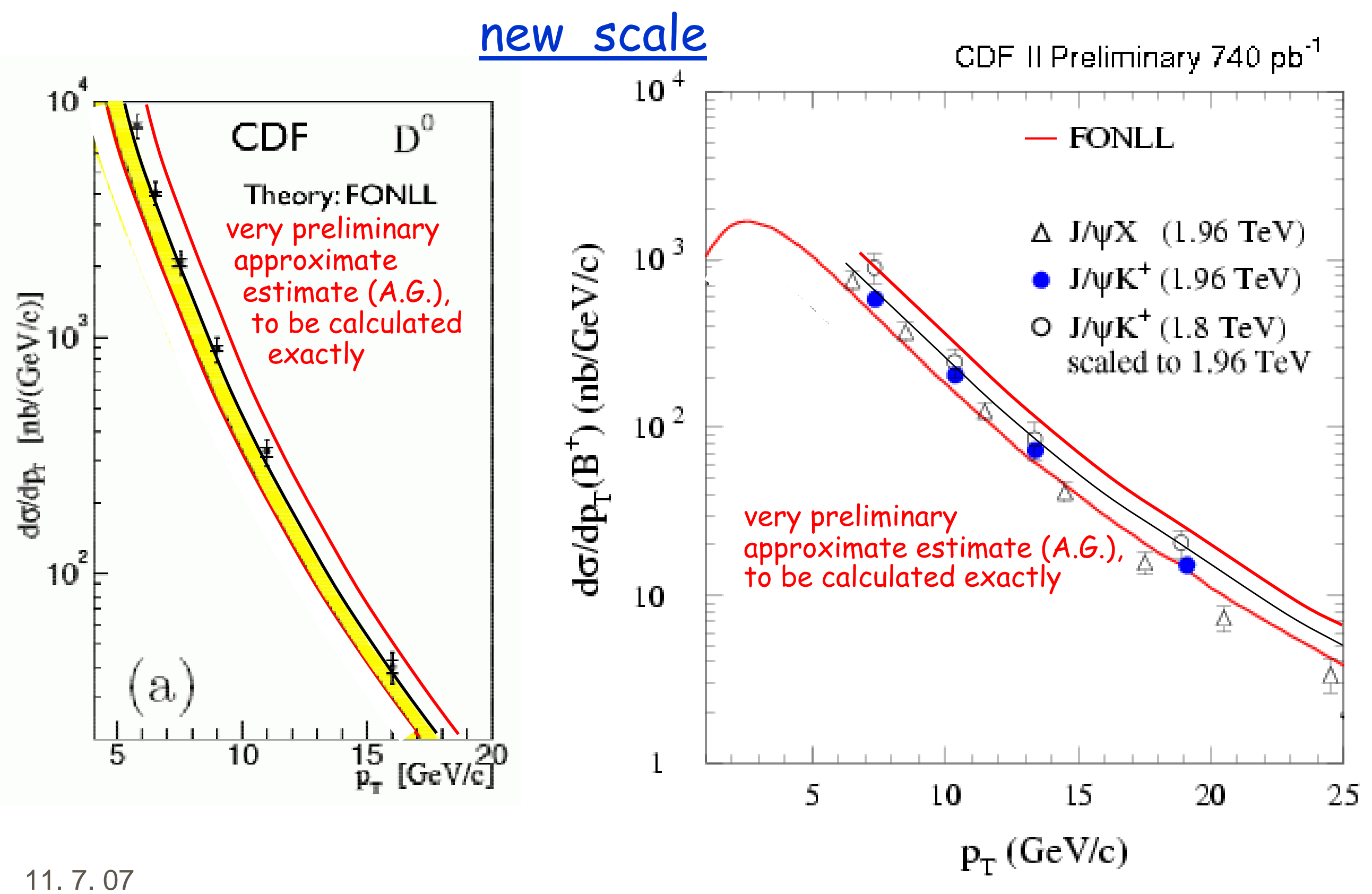




\section{beauty at Spp̄S, \\ $b+c$ at RHIC}

standard scale
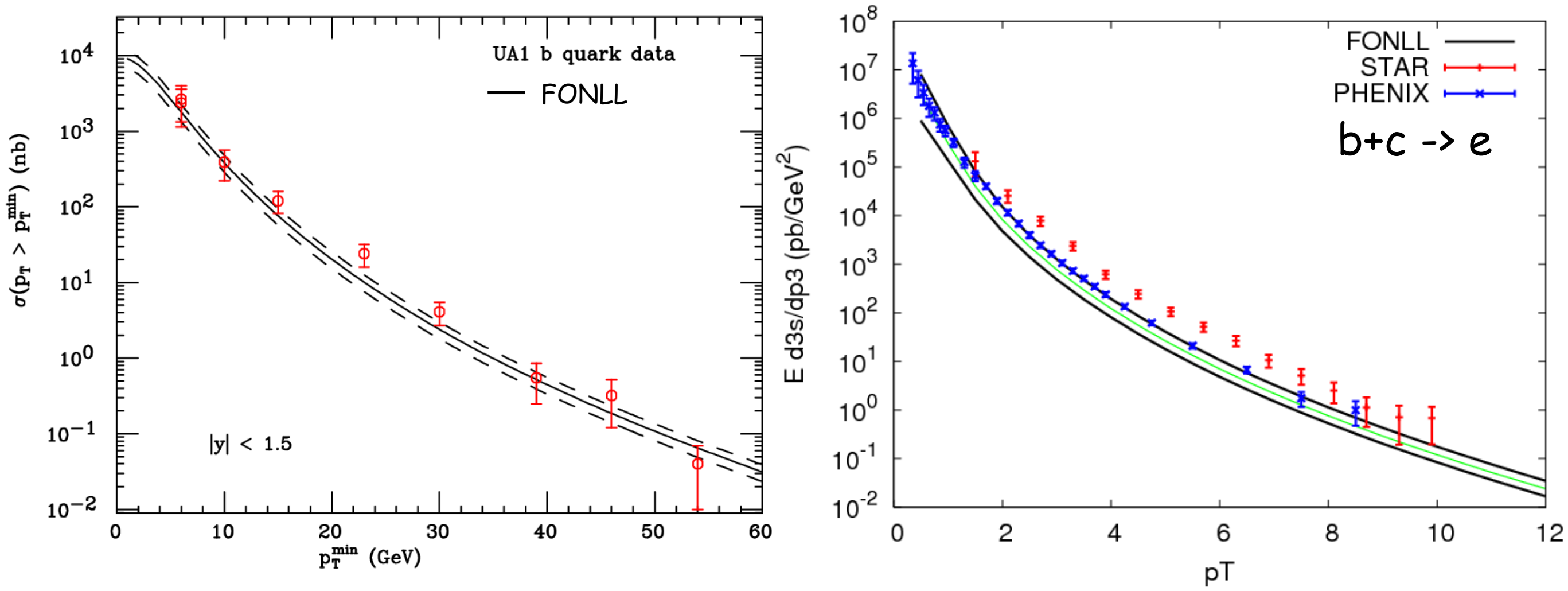


\section{beauty at Spp̄S,}

\section{$b+c$ at RHIC}

\section{new scale}
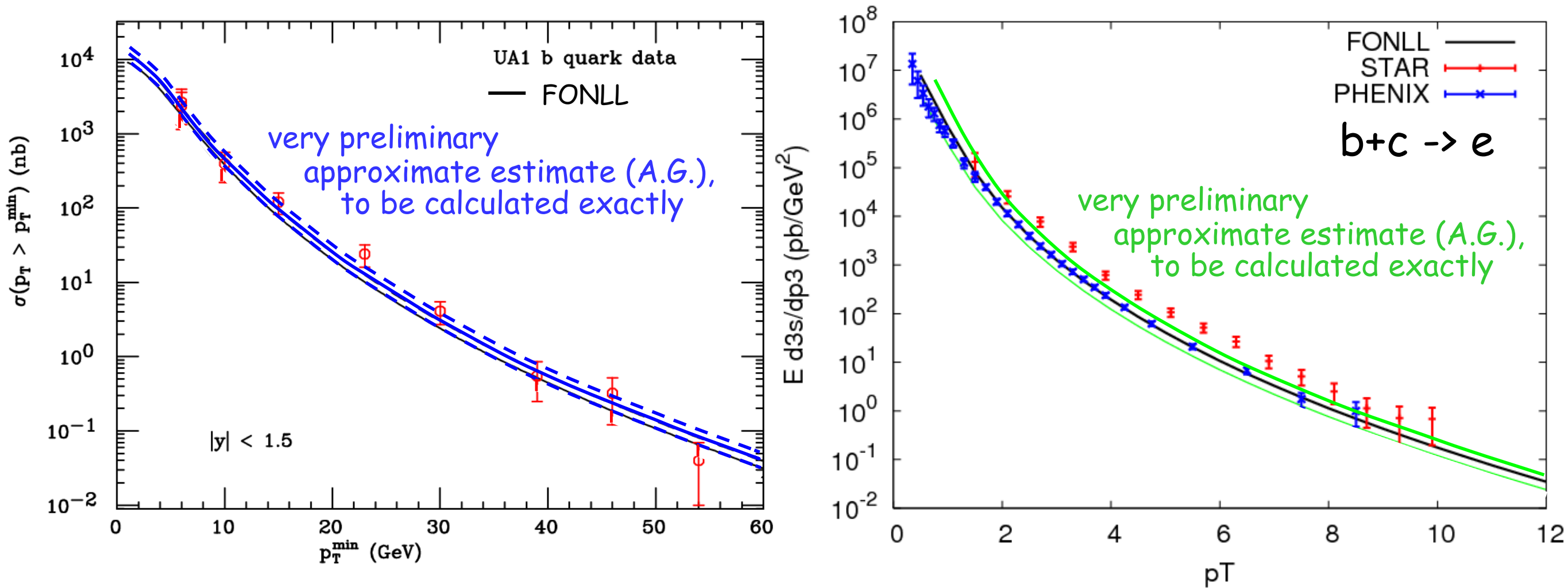


\section{top at Tevatron,}

\section{charm at HERA}

\section{standard scale}
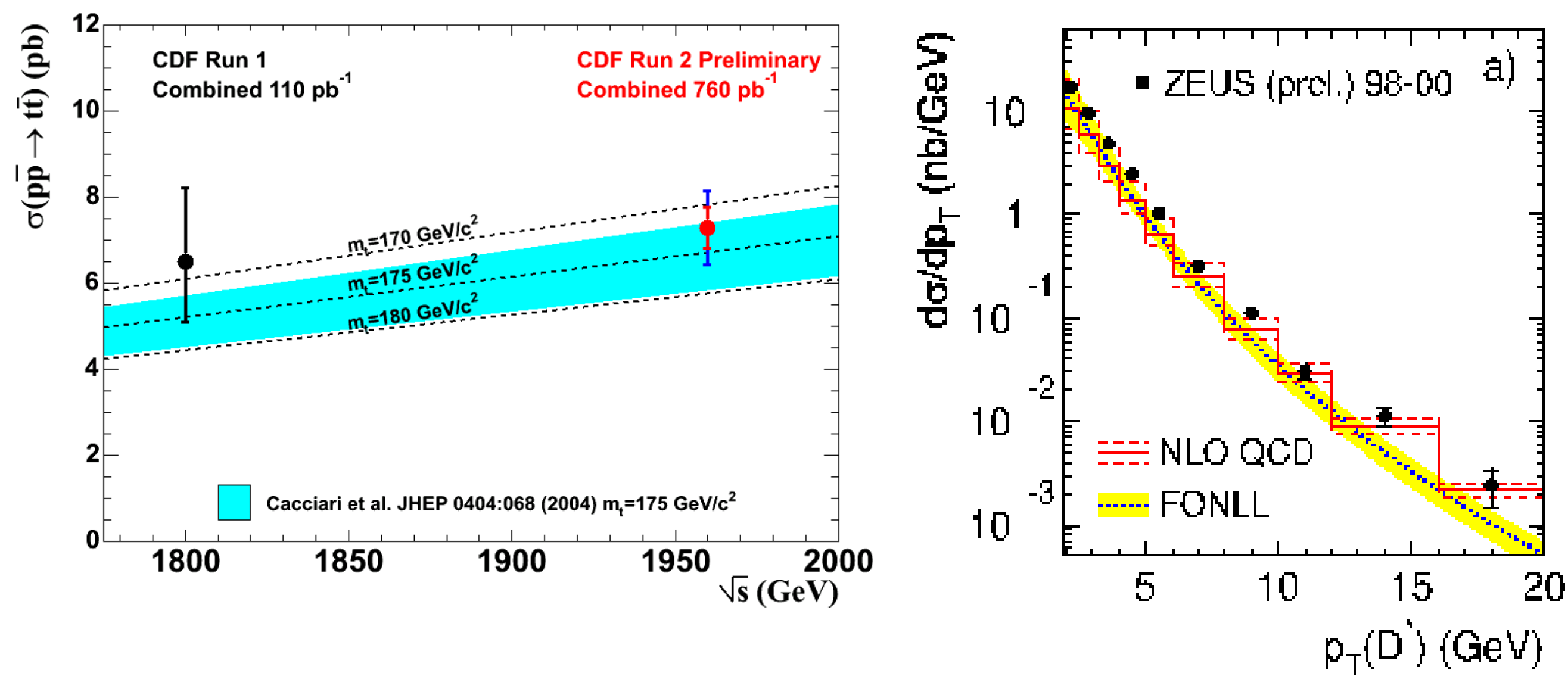


\section{top at Tevatron,}

\section{charm at HERA}

\section{new scale}
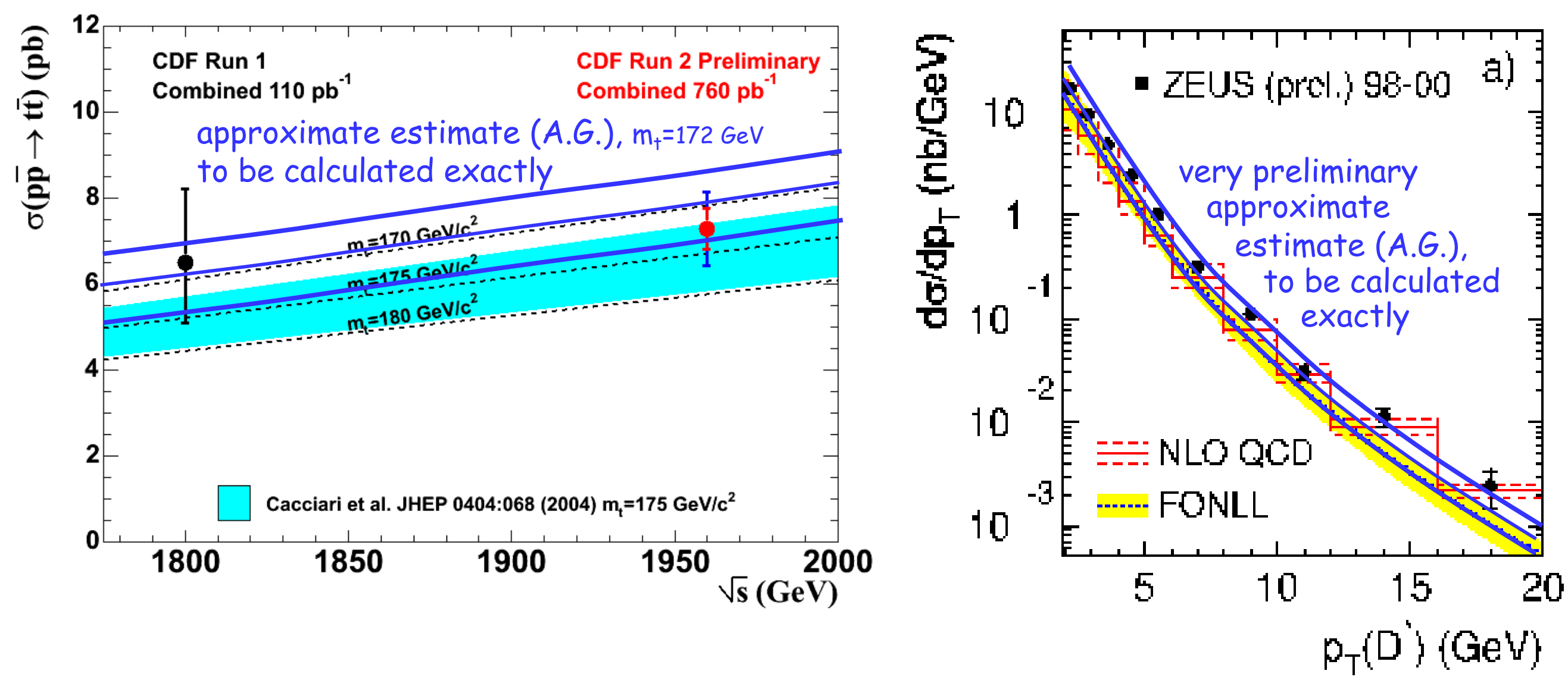


\section{Incl. Jets at HERA}

PHP

\section{standard scale}
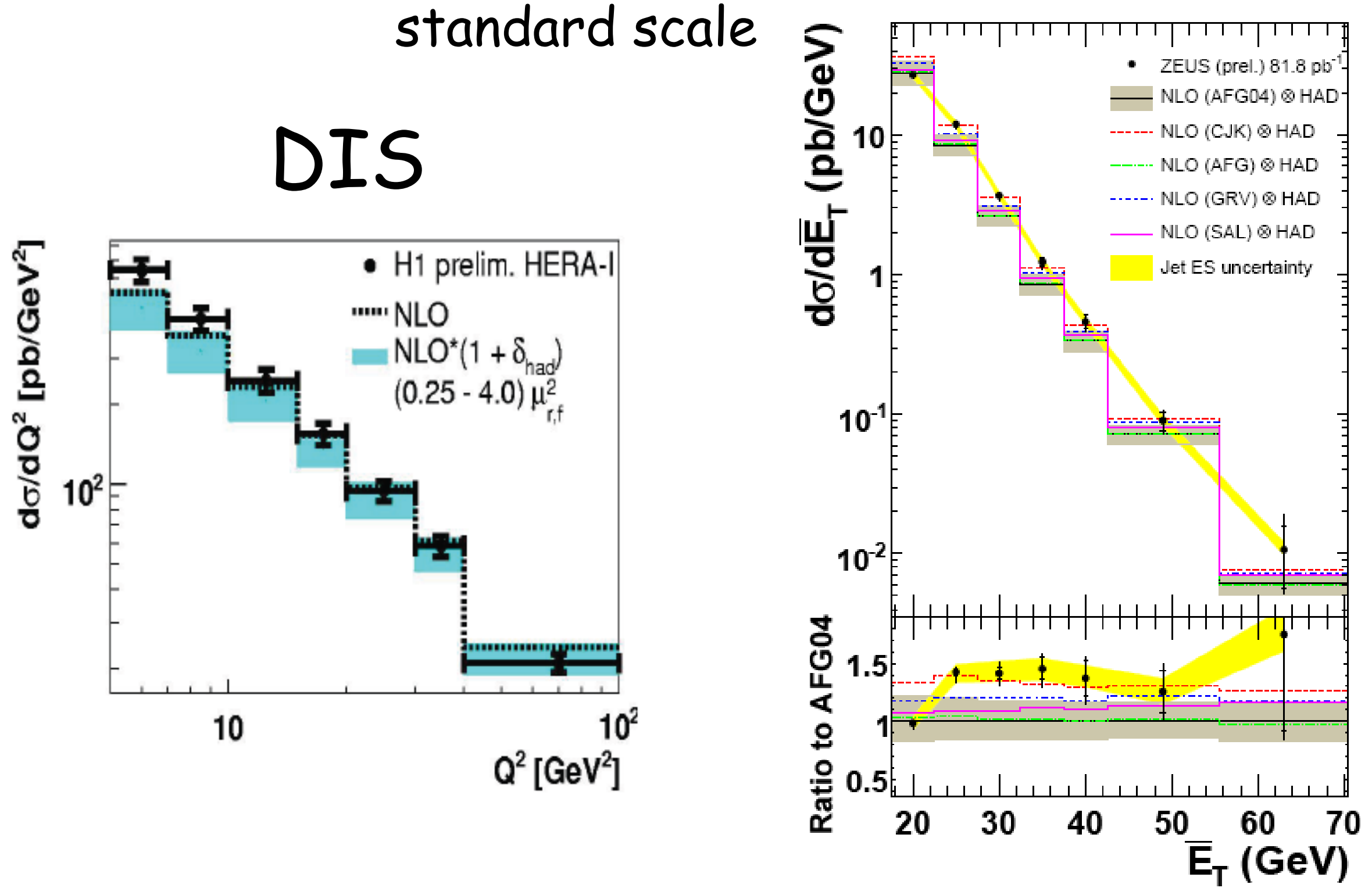


\section{Incl. Jets at HERA}

PHP

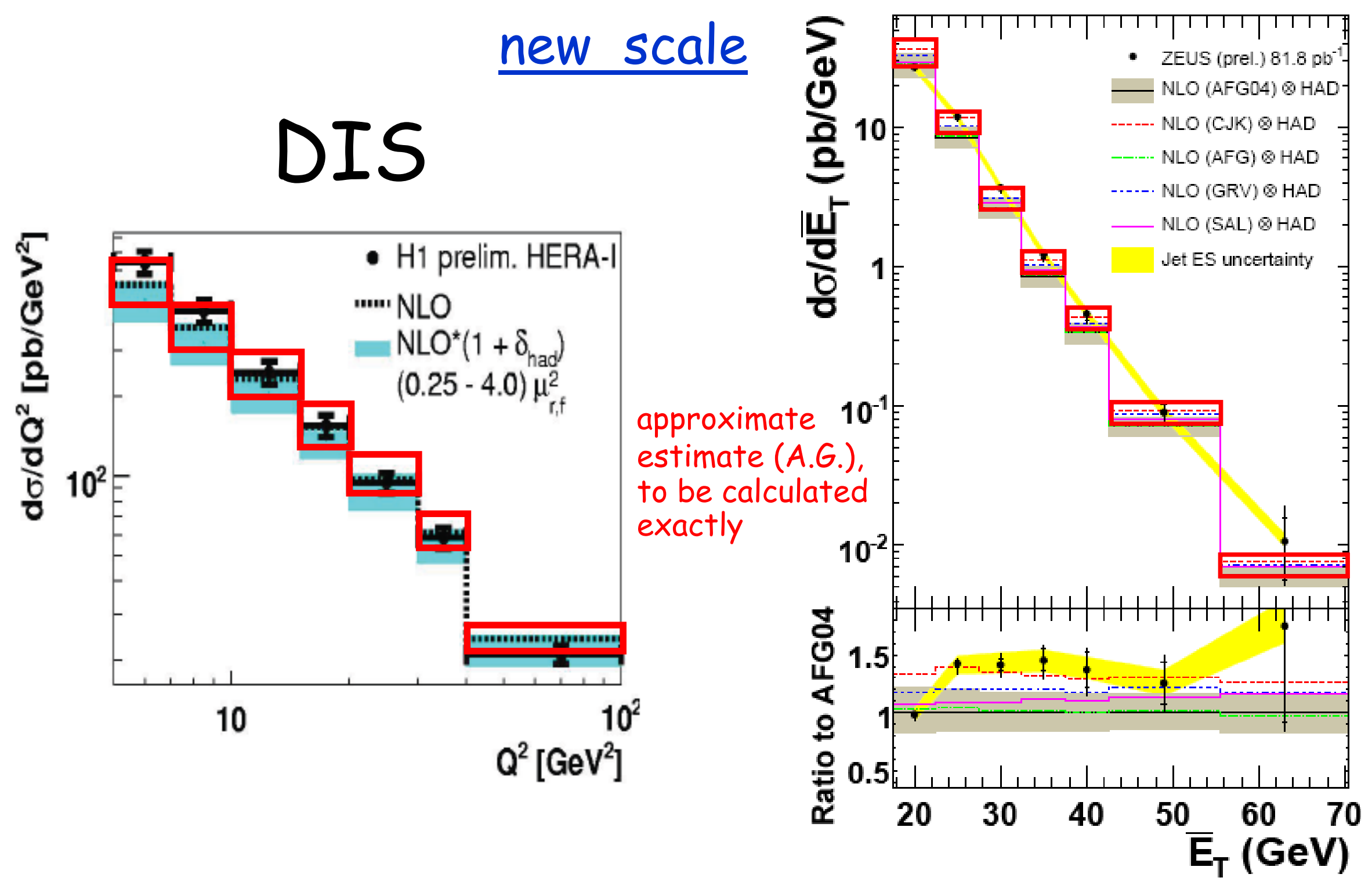




\section{Beauty in photoproduction: standard}

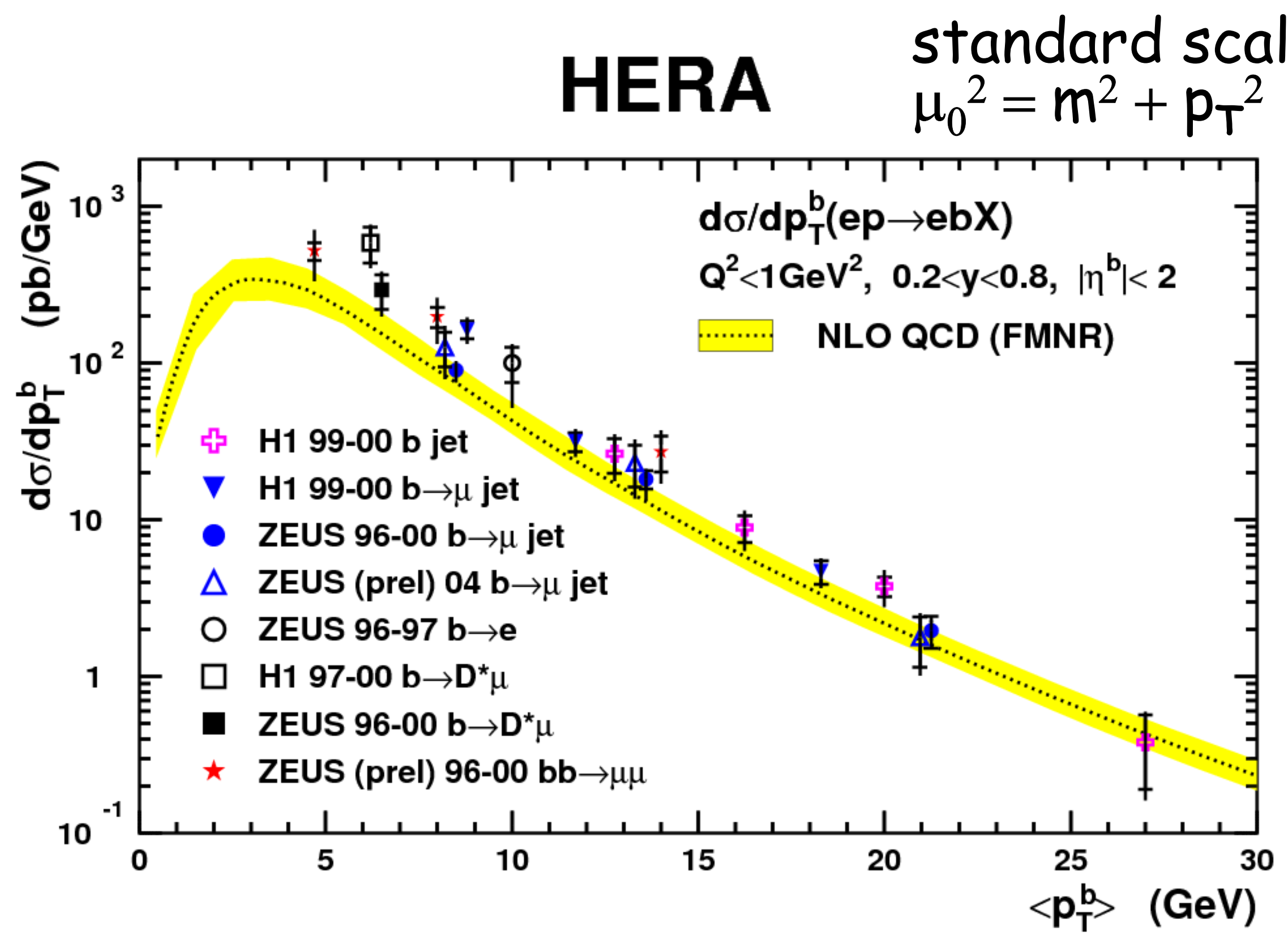




\section{Beauty in PHP: new reference scale}

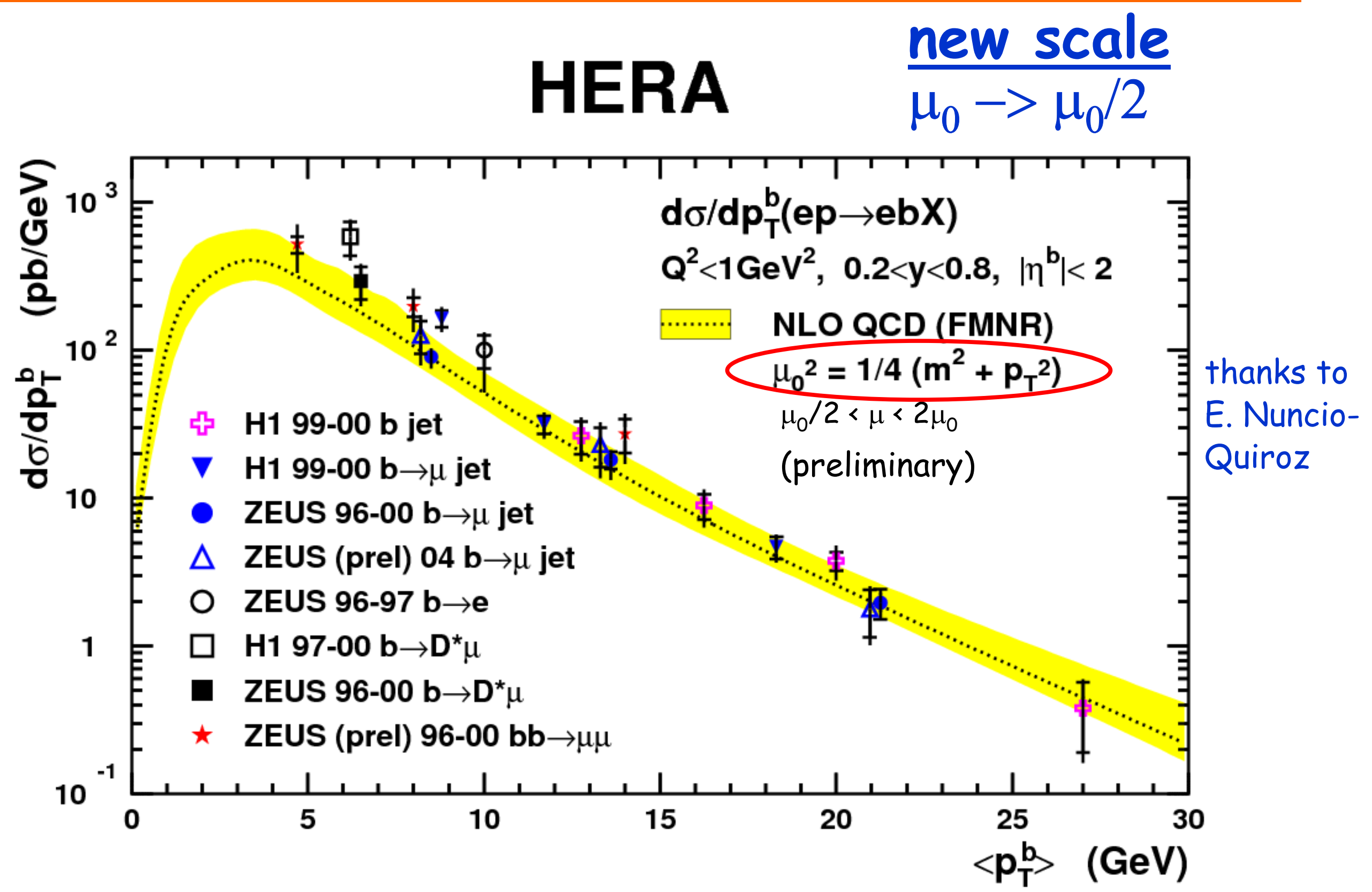




\section{Conclusion/Plea: either dedicated study, or}

propose, from now on, to use default QCD scale $\mu_{0} / 2$ for all heavy flavour (and other?) NLO cross section predictions at HERA and elsewhere, including LHC scale variation by factor 2 seems reasonable

some people are doing this already:

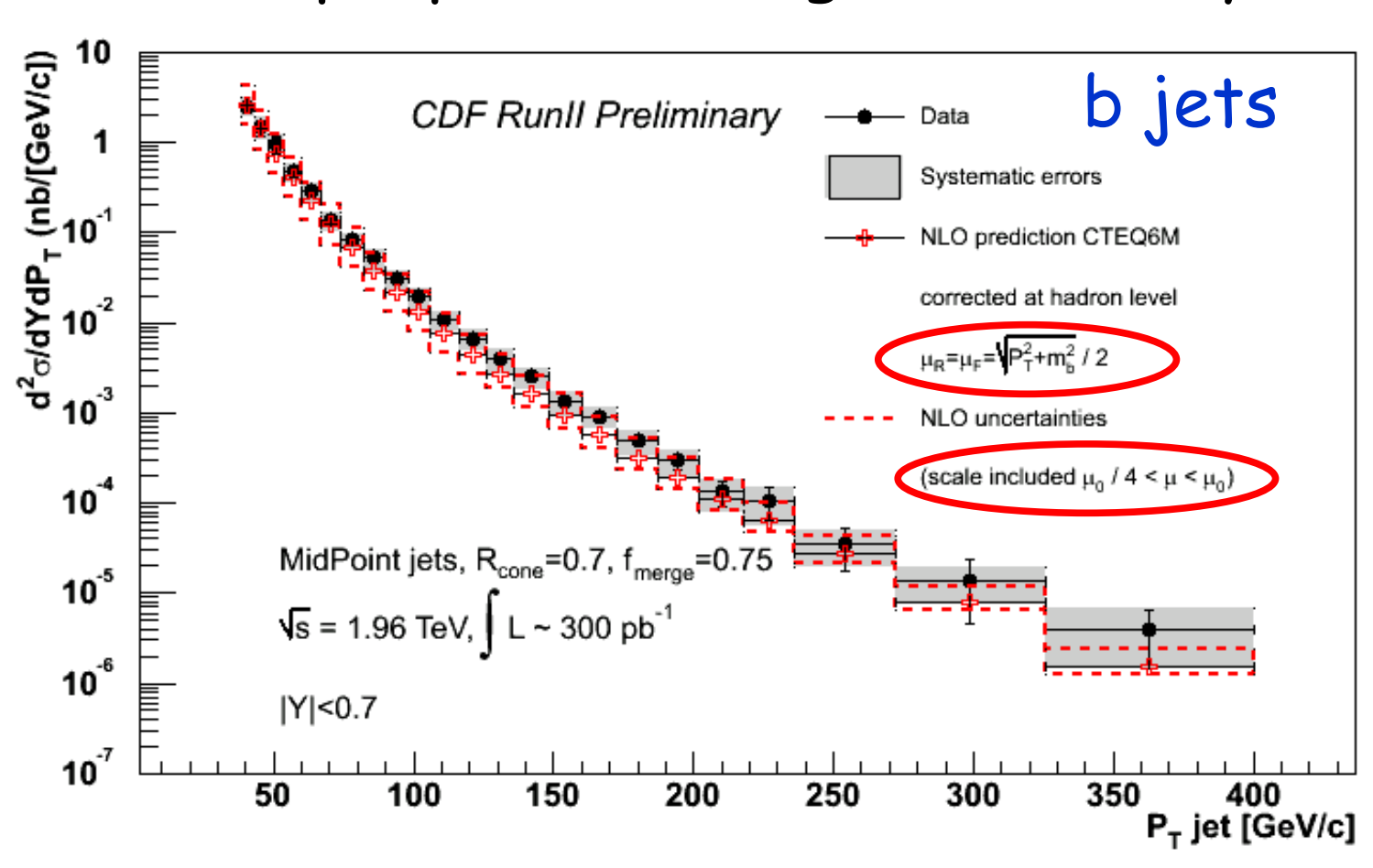

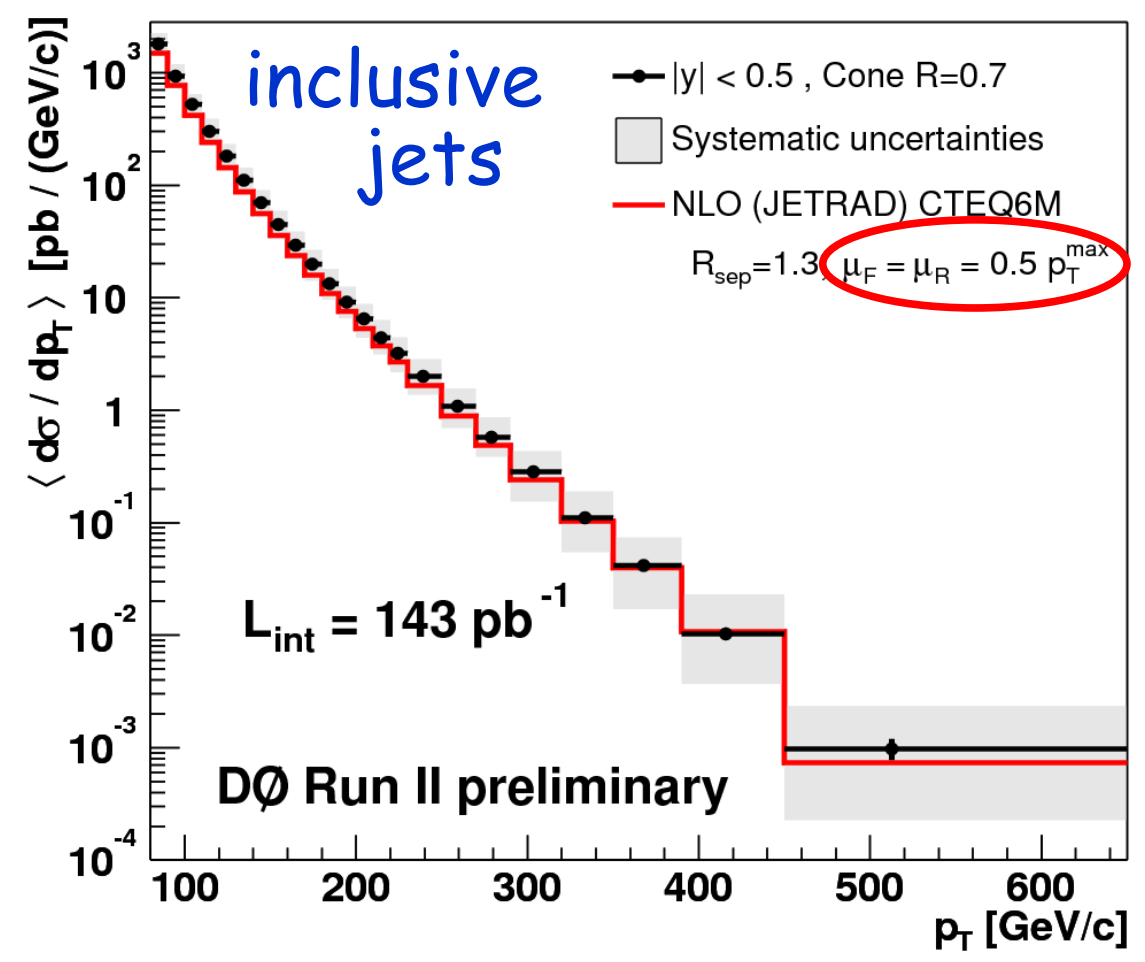




\section{also at HERA}

see also talk G. Grindhammer

\section{EPJ C44 (2005) 183: Multijet-Production in DIS}

\section{ZEUS}

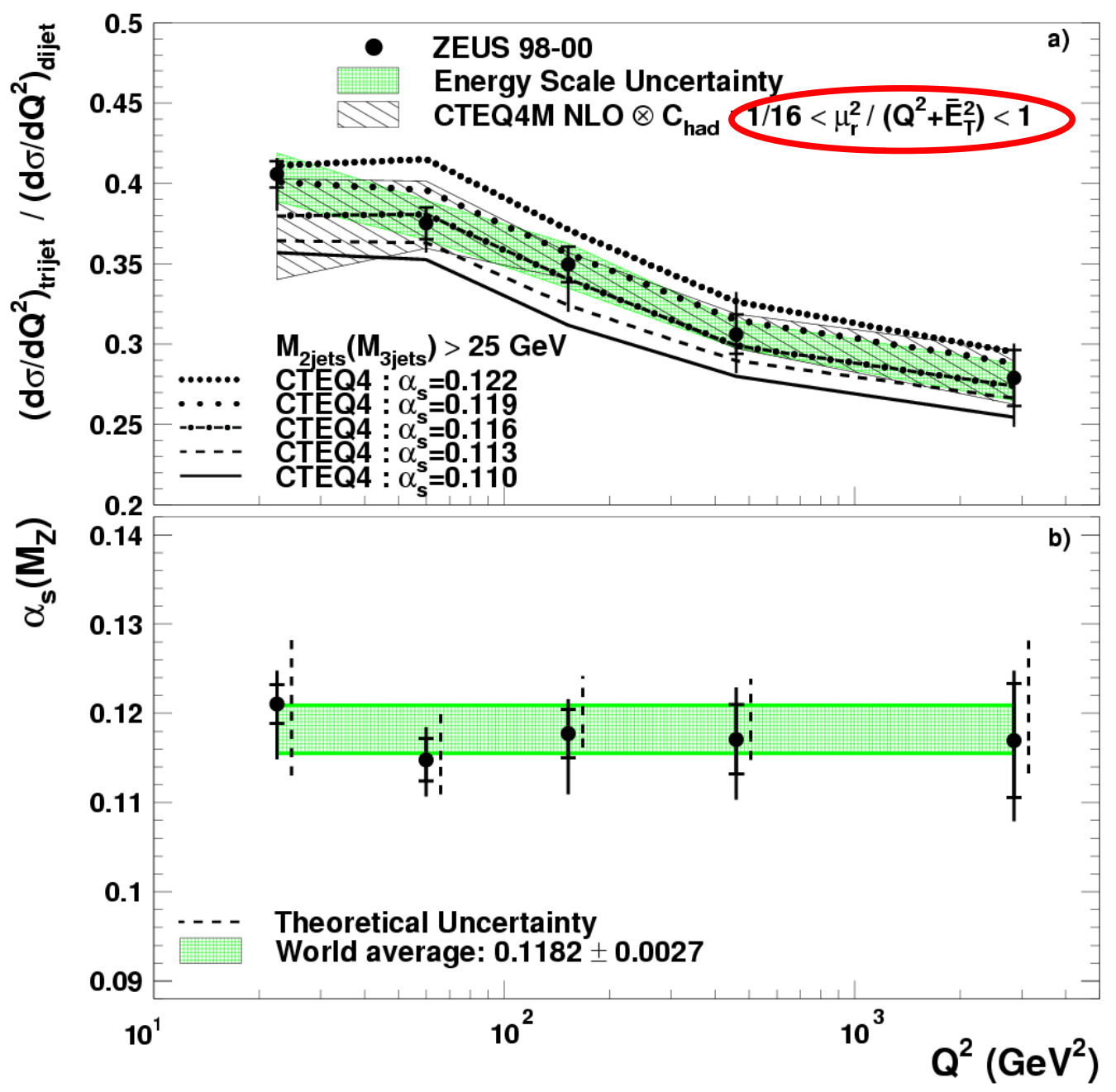




\section{BUT: Heavy Flavours in $\gamma \gamma$ at LEP?}

details on data: talk Finch.

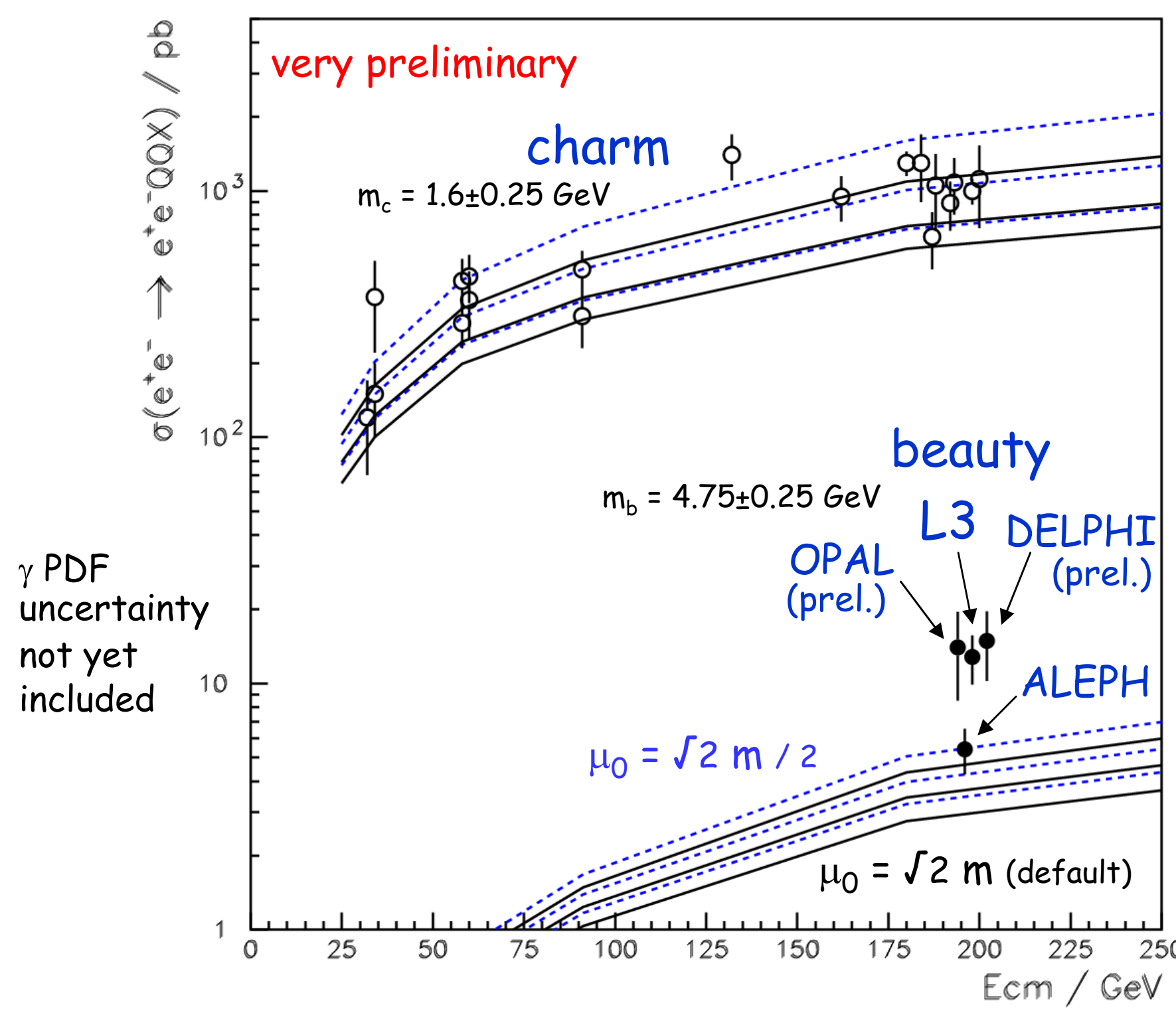

mainly

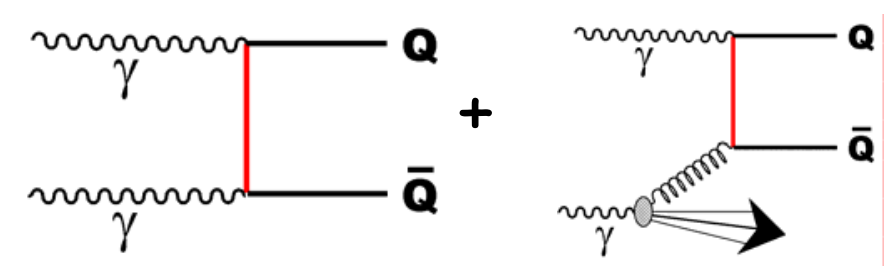

predictions adapted from

Drees et al., PLB 306 (1993) 371

changing scale $\mu_{0} \rightarrow \mu_{0} / 2$ helps, but not much (LO process is pure QED) 


\section{How well do we understand the quark/photon coupling?}




\section{investigate very tentative idea:}

Interference of QED with nonperturbative QCD?? Amplitudes (HERA):

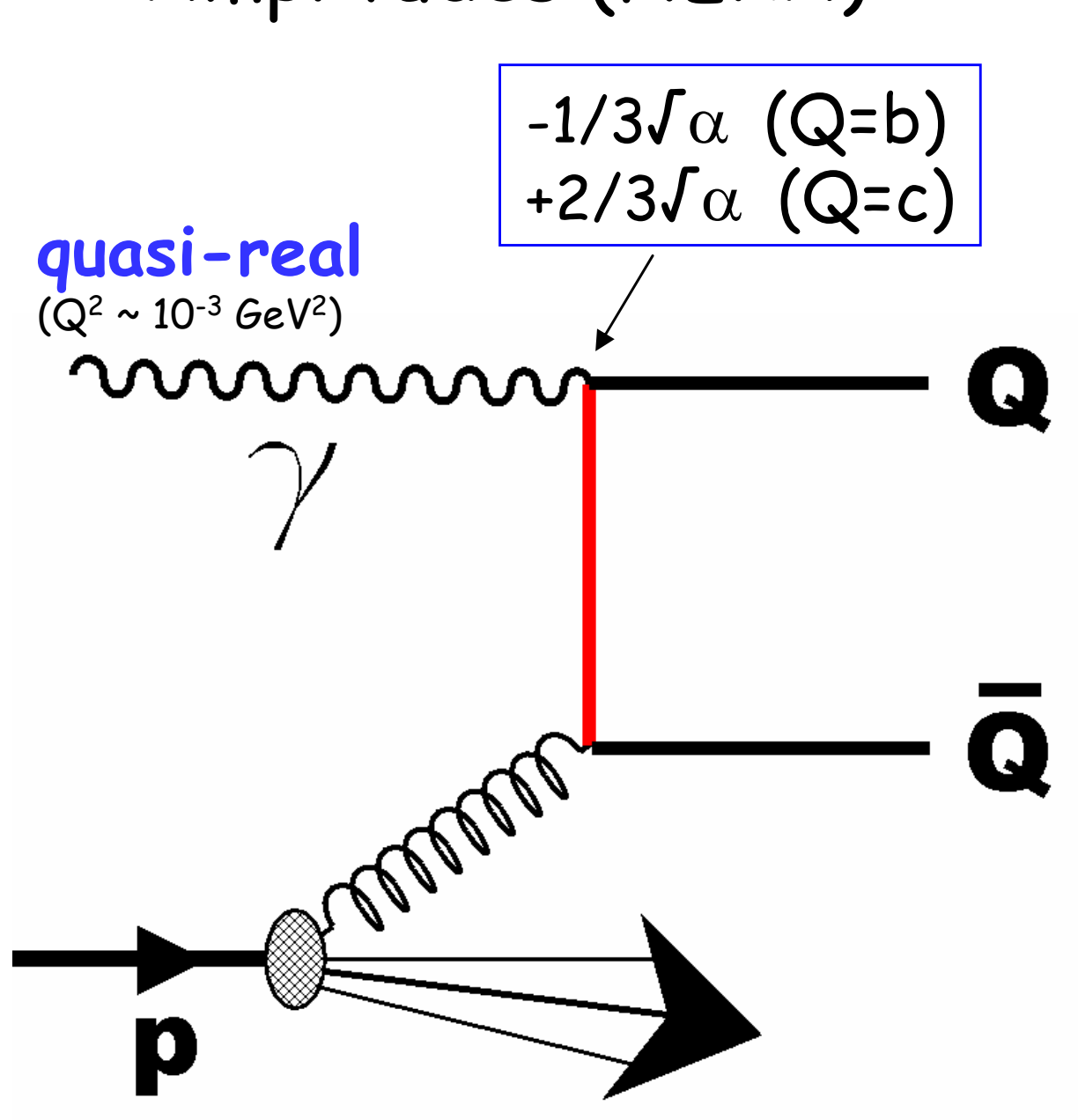

colourless system of 3 quasi-real gluons
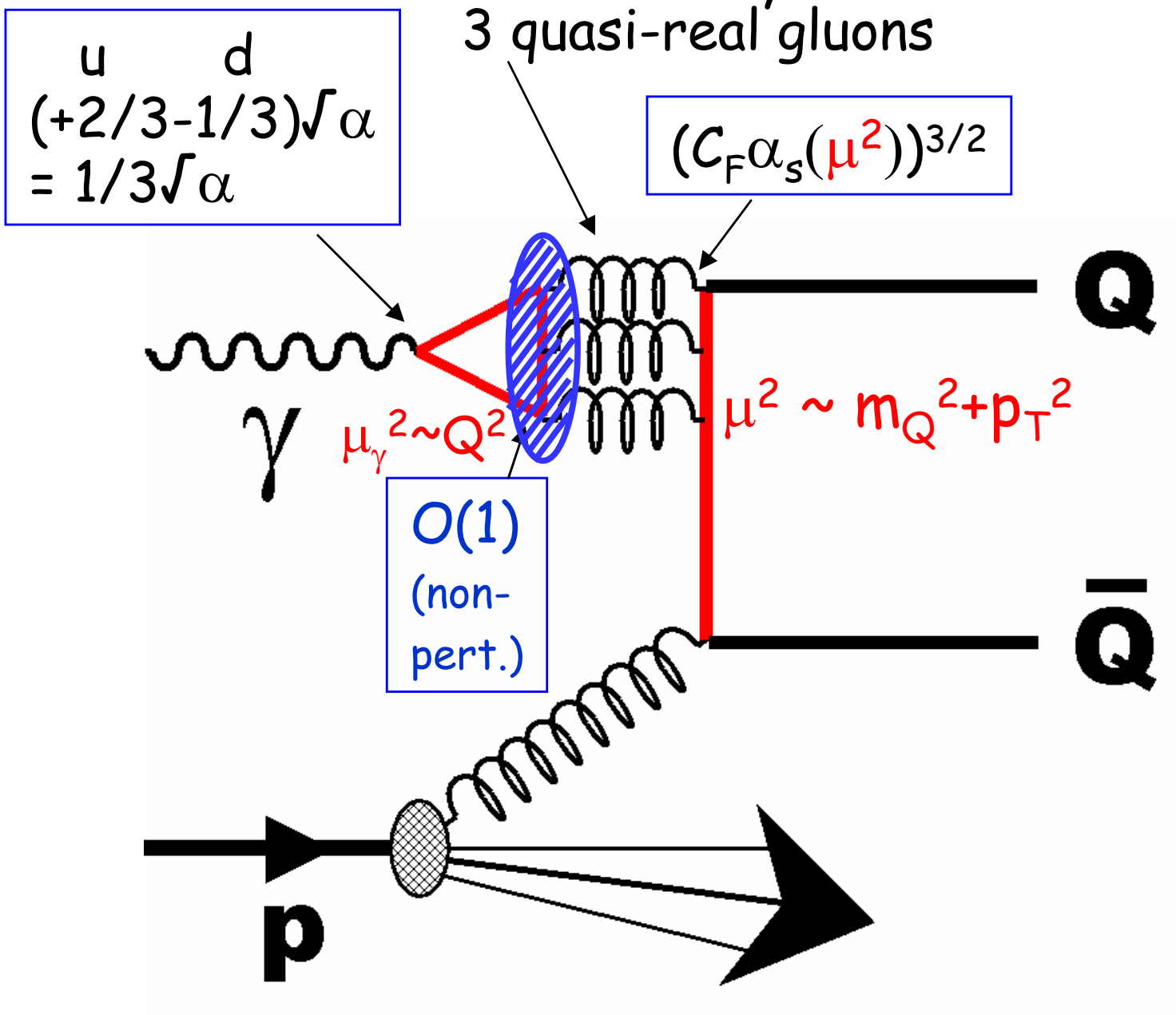
Interference of QED with nonperturbative QCD?

amplitudes for effective photon coupling:

$b \sim-1 / 3\left(1+\kappa\left(C_{F} \alpha_{S}\left(\mu^{2}\right)\right)^{3 / 2}\right) \quad \mu^{2} \sim m_{Q}^{2}+p_{T}^{2} \sim 2 m_{Q}^{2}$ cancellation $\uparrow$

$\sim 0.14$

$\alpha_{s}\left(2 m_{b}^{2}\right) \approx 0.20$

$c \sim 2 / 3\left(1-\frac{1}{2} \kappa\left(C_{F} \alpha_{S}\left(\mu^{2}\right)\right)^{3 / 2}\right)$

$\sim 0.22$

$\alpha_{s}\left(2 m_{c}^{2}\right) \approx 0.27$

$\left(\sim 0.15\right.$ for $\left.\mathrm{p}_{\mathrm{Tc}} \sim \mathrm{m}_{\mathrm{b}}\right)$

where $K=$ unknown complex factor of non-perturbative origin (of order 1 )

example: arbitrarily assume $K \approx 1$ (real)

$\Rightarrow$ effective $\gamma b$ coupling enhanced by factor $(1+0.14)^{2}=1.30$

$\Rightarrow$ effective $\gamma c$ coupling reduced by factor $\left(1-\frac{1}{2} \times 0.22\right)^{2}=0.79$ 


\section{Heavy Flavours in $\gamma \gamma$ at LEP, revisited}

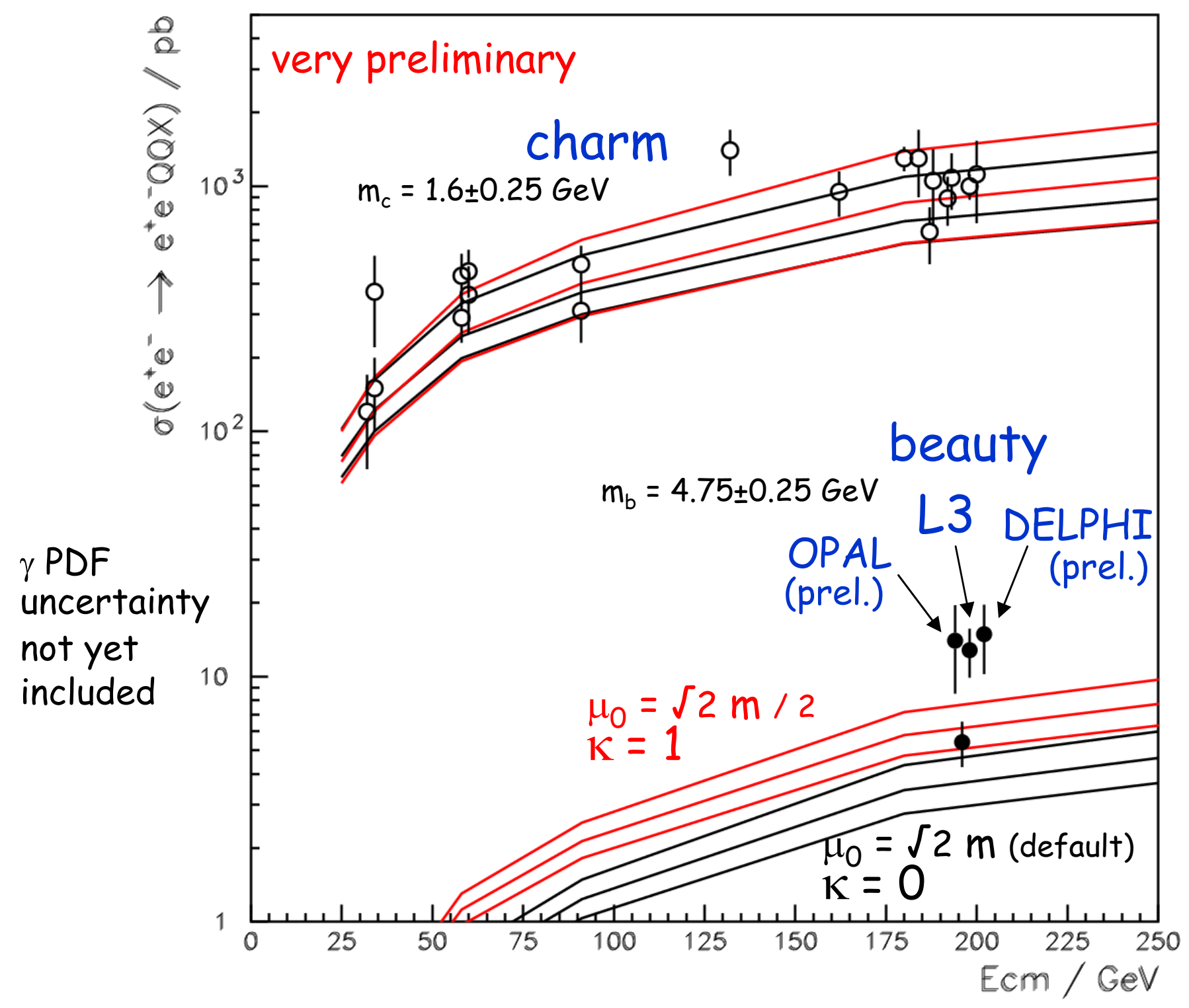

scale $\uparrow$ and coupling $\downarrow$ changes cancel

standard predictions $(\kappa=0)$ from Drees et al., PLB 306 (1993) 371

scale $\uparrow$ and coupling $\uparrow$ changes add 


\section{Beauty in photoproduction: standard}

reminder:

$$
\text { HERA } \begin{aligned}
& \mu_{0}^{2}=\mathrm{m}^{2}+\mathrm{PT}^{2} \\
& \text { standard photon coupling }
\end{aligned}
$$

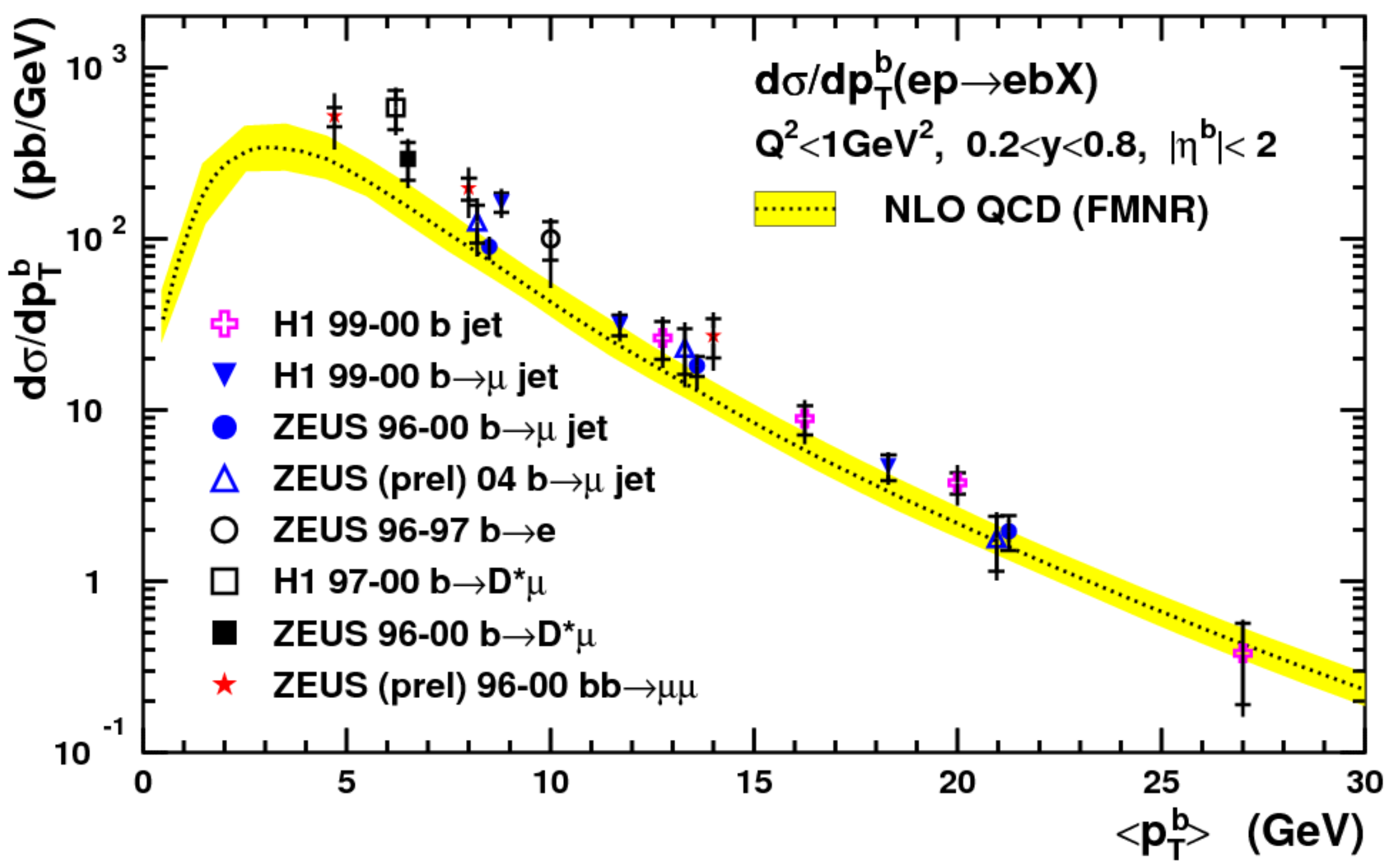




\section{Beauty in photoproduction: new scale}

reminder:

$$
\begin{array}{ll}
\text { HERA } & \mu_{0}->\mu_{0} / 2 \\
\text { standard photon coupling }
\end{array}
$$

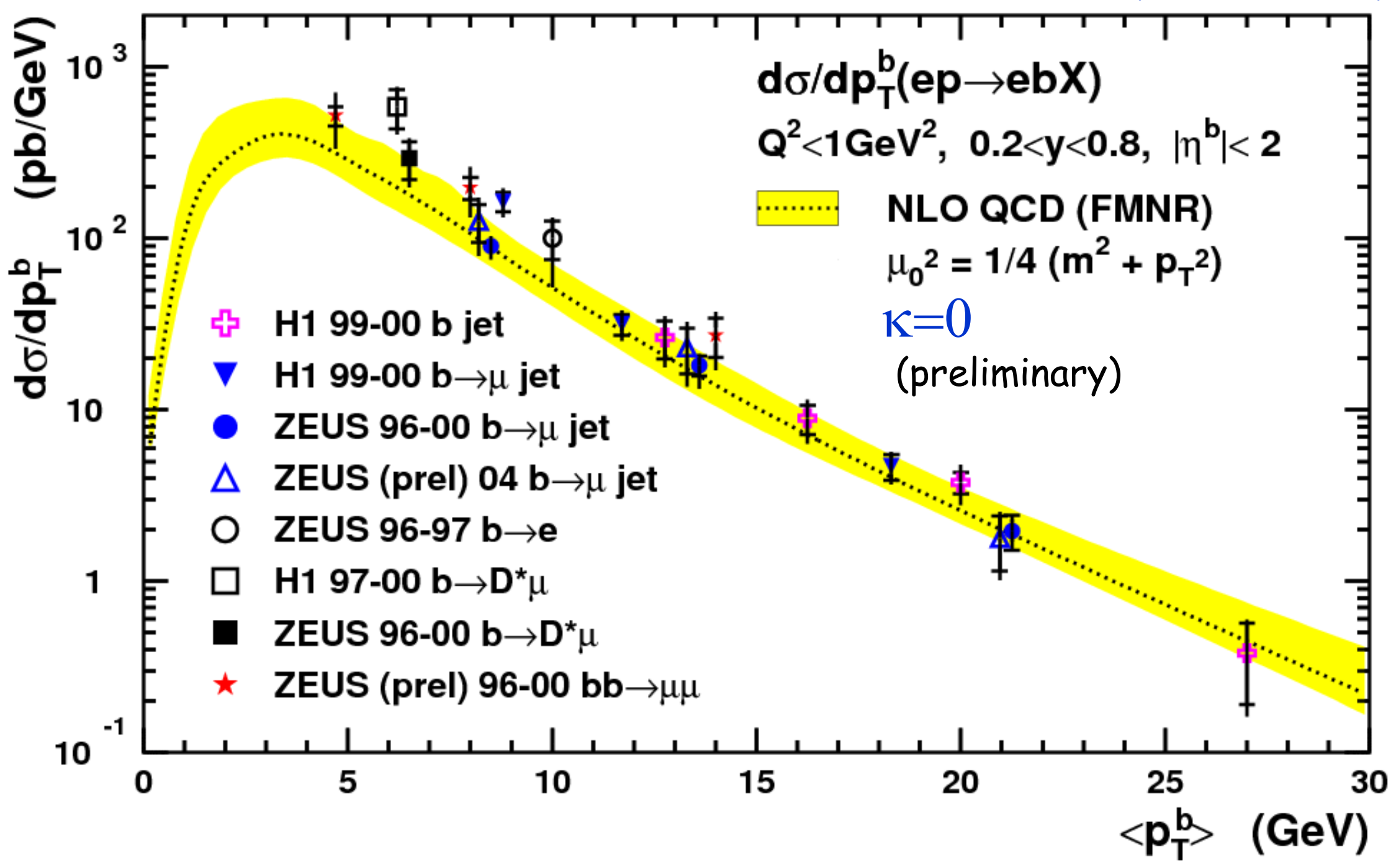




\section{Beauty in photoproduction: non-standard}

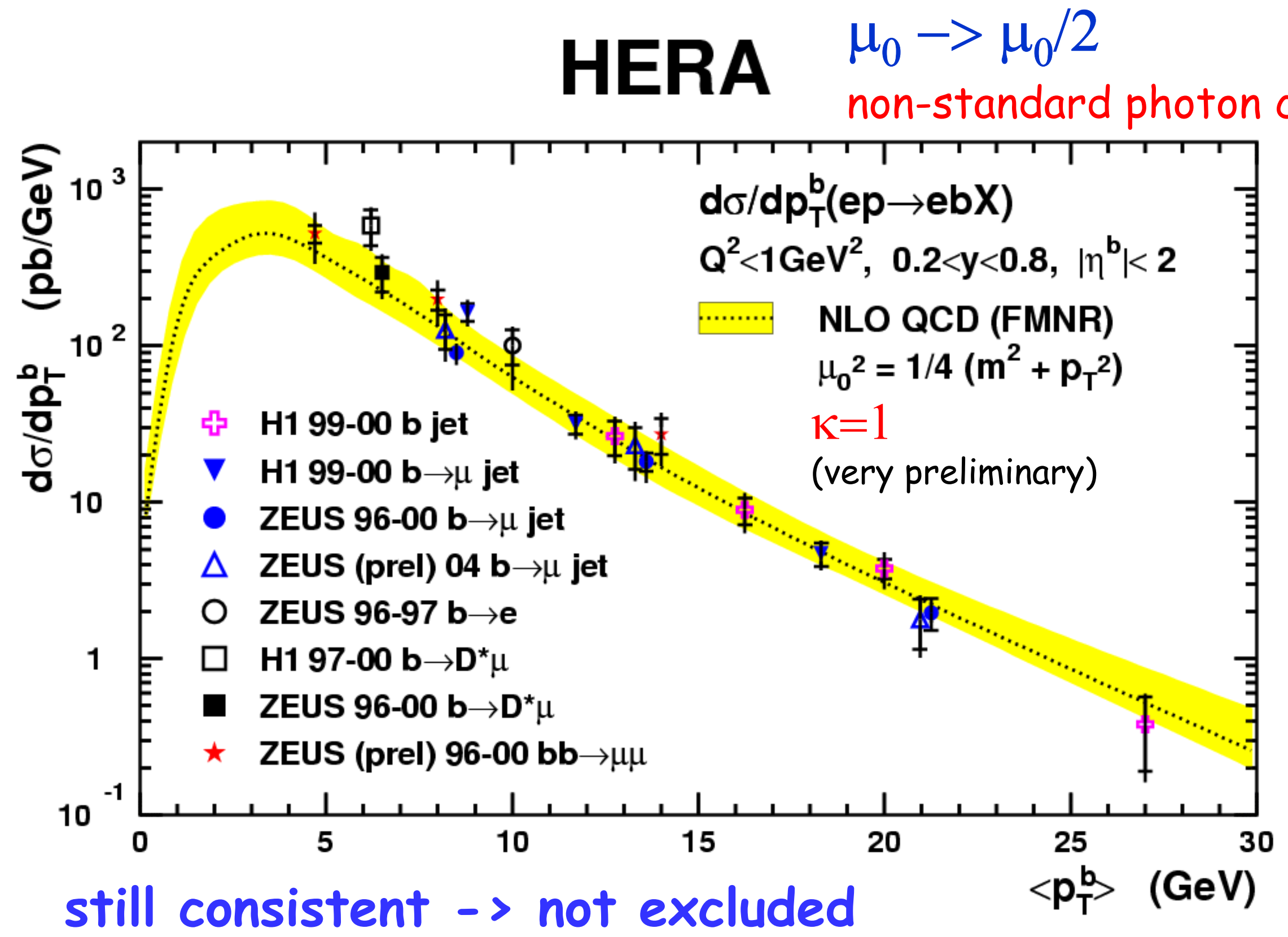




\section{compatibility with other measurements?}

purely hadronic processes (Tevatron, LHC) unaffected
purely weak processes (W/Z at Tevatron, CC at HERA) unaffected

change of coupling only relevant for (quasi-)real photons $\left(Q^{2}>1 \mathrm{GeV}^{2} \rightarrow\right.$ perturbative $\rightarrow \kappa \ll 1 \rightarrow$ very small/negligible) no significant change for DIS at HERA, $e^{+} e^{-} \rightarrow q \bar{q} X$

reduction of charm photoproduction at HERA, qualitatively compensated by increase due to $\mu \rightarrow \mu / 2$ (to be checked quantitatively)

for hard scale $\mu$ > $m_{q}$, modifications for up and down quark contributions cancel exactly in leading order $\Rightarrow u, d ; s, c$ cancel no net effect for 2,4,6 final state flavours (e.g. $N_{F}=4 \gamma p$ fixed target) some increase ( $2 \%$ ) in PHP high $E_{T}$ jet production at HERA, high $E_{T}$ single photon production at Tevatron (5=3-2 flavours, b only)

should affect e.m. branching ratios, e.g. D*->Dy (but not electroweak ones like $b->s \gamma$, since $W \gamma$ coupling unaffected)

other processes? 


\section{Summary and conclusions}

- Beauty cross sections at HERA and elsewhere in reasonable agreement with perturbative $Q C D$ predictions (but often above "central" prediction).

HERA

- Phenomenological arguments (independent of data) suggest shift in choice of "optimal" renormalization/factorization scales to walf their "standard" values

$\Rightarrow$ good agreement with many different data sets

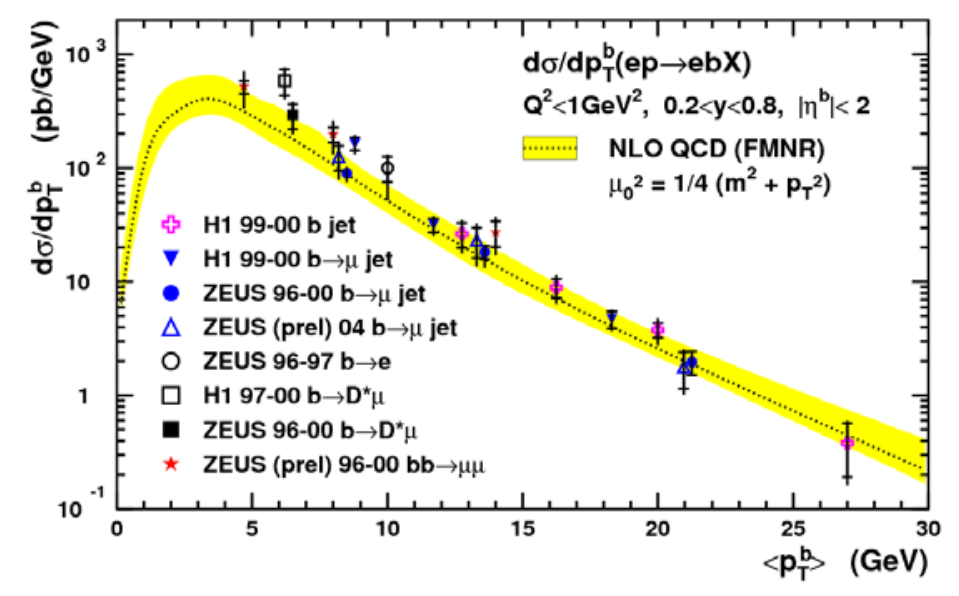

Plea to make this the new default, whenever a dedicated study is absent, in particular before claiming disagreement between data and NLO QCD. up/down scale variation by factor 2 looks OK. (theorists who do not like this: please provide NNLO calculations!)

b "excess" of $\gamma \gamma$ at LEP? $\Rightarrow$ investigate modification of photon-quark coupling from interference with non-perturbative QCD? $\Rightarrow$ compatible with HERA beauty data. Compatibility with other measurements and theoretical constraints to be checked Important e.g. for $\alpha_{s}$ from PHP jets at HERA $\rightarrow$ hopefully exclude (confirm??) soon. 


\section{Backup slides}




\section{PQCD approximations}

assume one dominant hard scale:

Massive scheme: $\rightarrow m_{b}$

- b massive $p_{T}^{2}$

- neglects $\left[\alpha_{s} \ln \left(Q^{2} / m_{b}^{2}\right)\right]^{n}$

$\rightarrow$ Perturbative production:

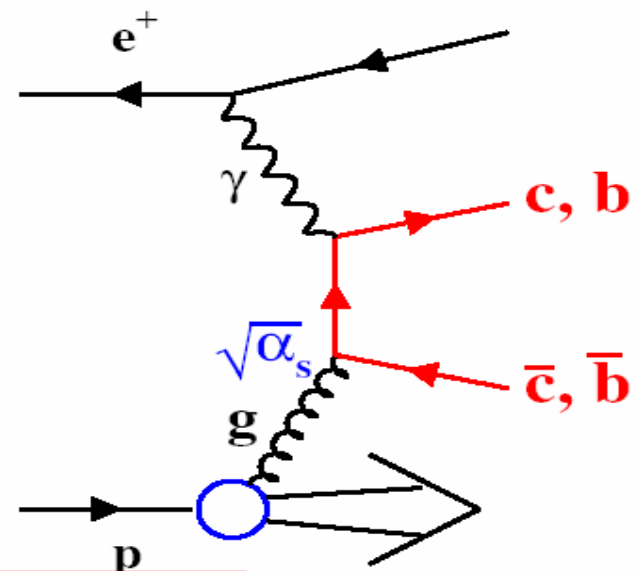

Massless scheme: $\rightarrow p_{T}, Q^{2}$

- b massless! $p_{T}^{2}$

- Resums $\left[\alpha_{s} \ln \left(Q^{2} / m_{b}^{2}\right)\right]^{n}$

$\rightarrow \mathbf{b}$ also in Proton and Photon!
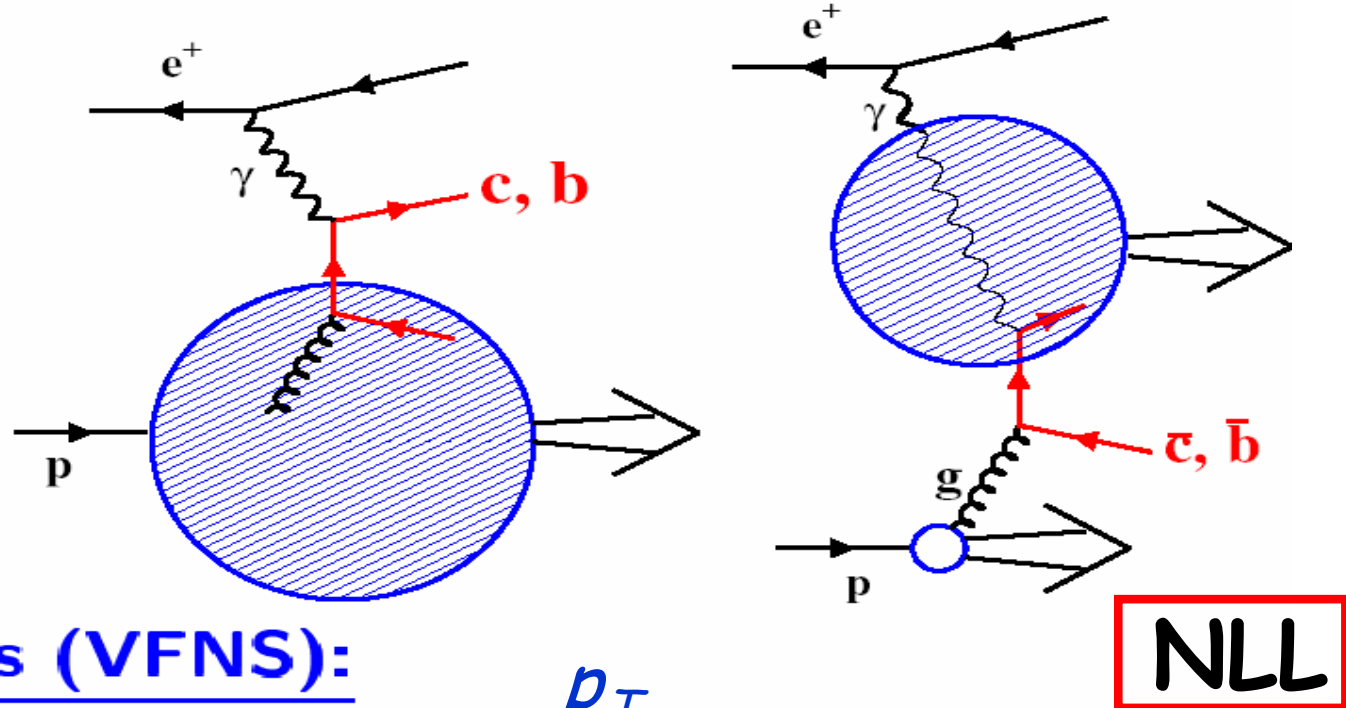

Variable schemes (VFNS):

$\rightarrow$ at small $Q^{2}$ massive, at large $Q^{2}$ massless

=FFNS

$p_{T}$ FONLL

$=$ ZM-VFNS alternative: kt-factorization
(GM)-VFNS 


\section{Beauty in photoproduction: summary}

bquark HERA

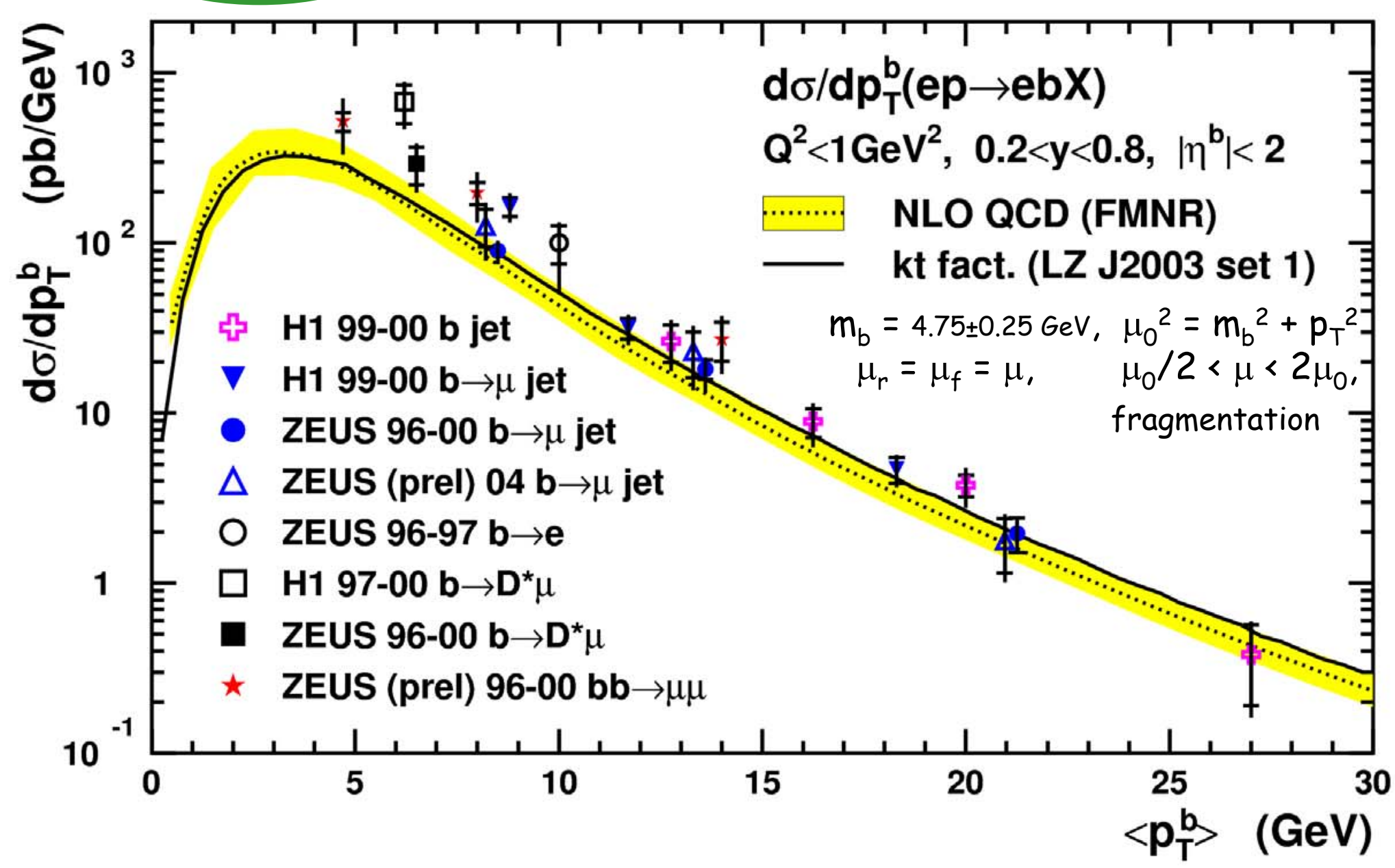

data/QCD:

reasonable agreement, but tendency data $>$ QCD at low $\mathrm{PT}_{\mathrm{T}}$

$k_{T}$ factoriz. and NLO predictions agree

theory uncert. underestimated?

FONLL (VFNS) prediction not yet available, should it help? 


\section{famous $b$ cross sections at the Tevatron}

(a bit of history)

combination of appropriate fragmentation, NLL corrections, + many smaller experimental and theoretical issues

(Cacciari et al.)
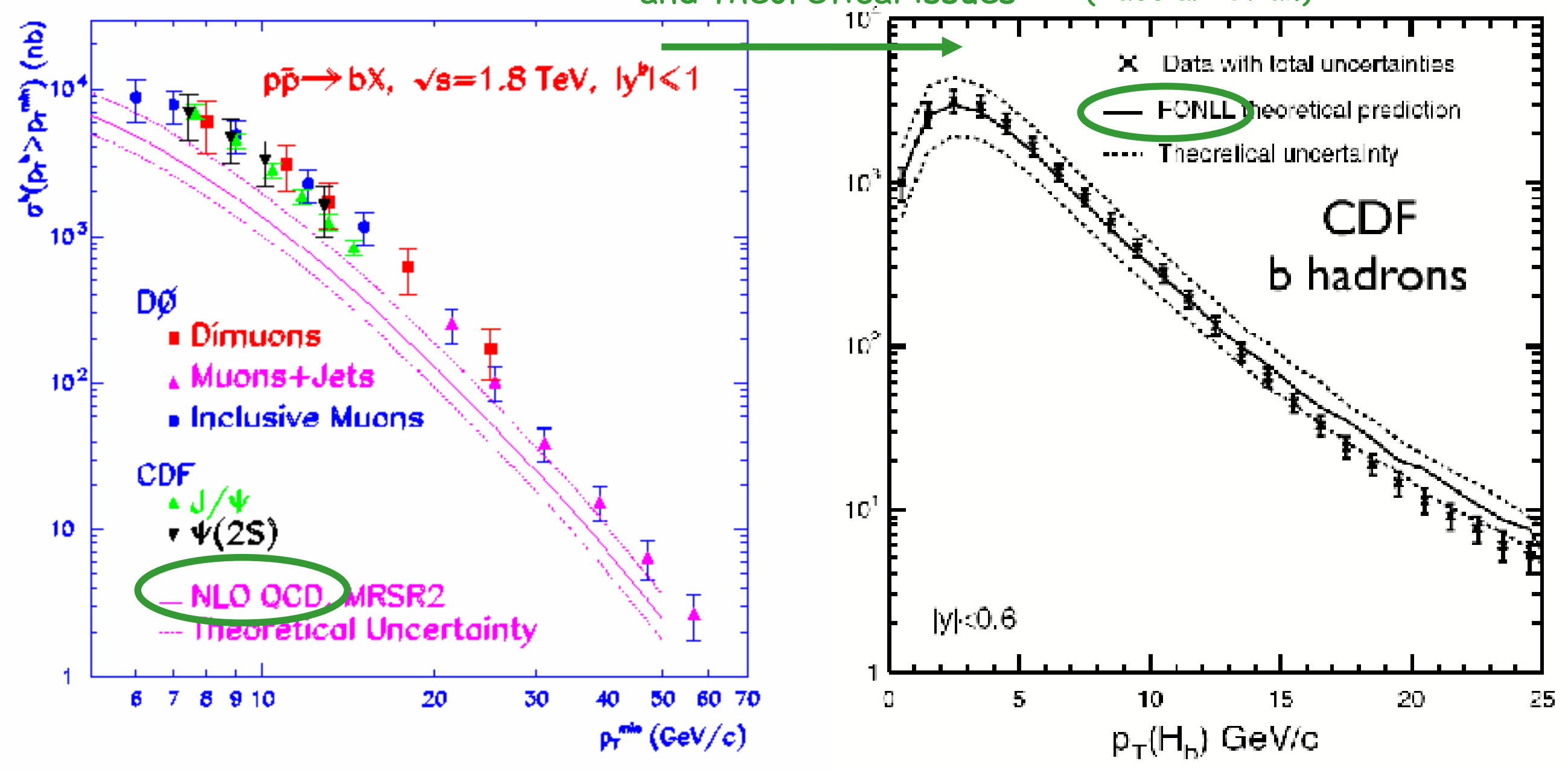

problem also for HERA? could one have done better? 


\section{$b$ cross sections at UA1 (630 GeV p $\bar{p})$}
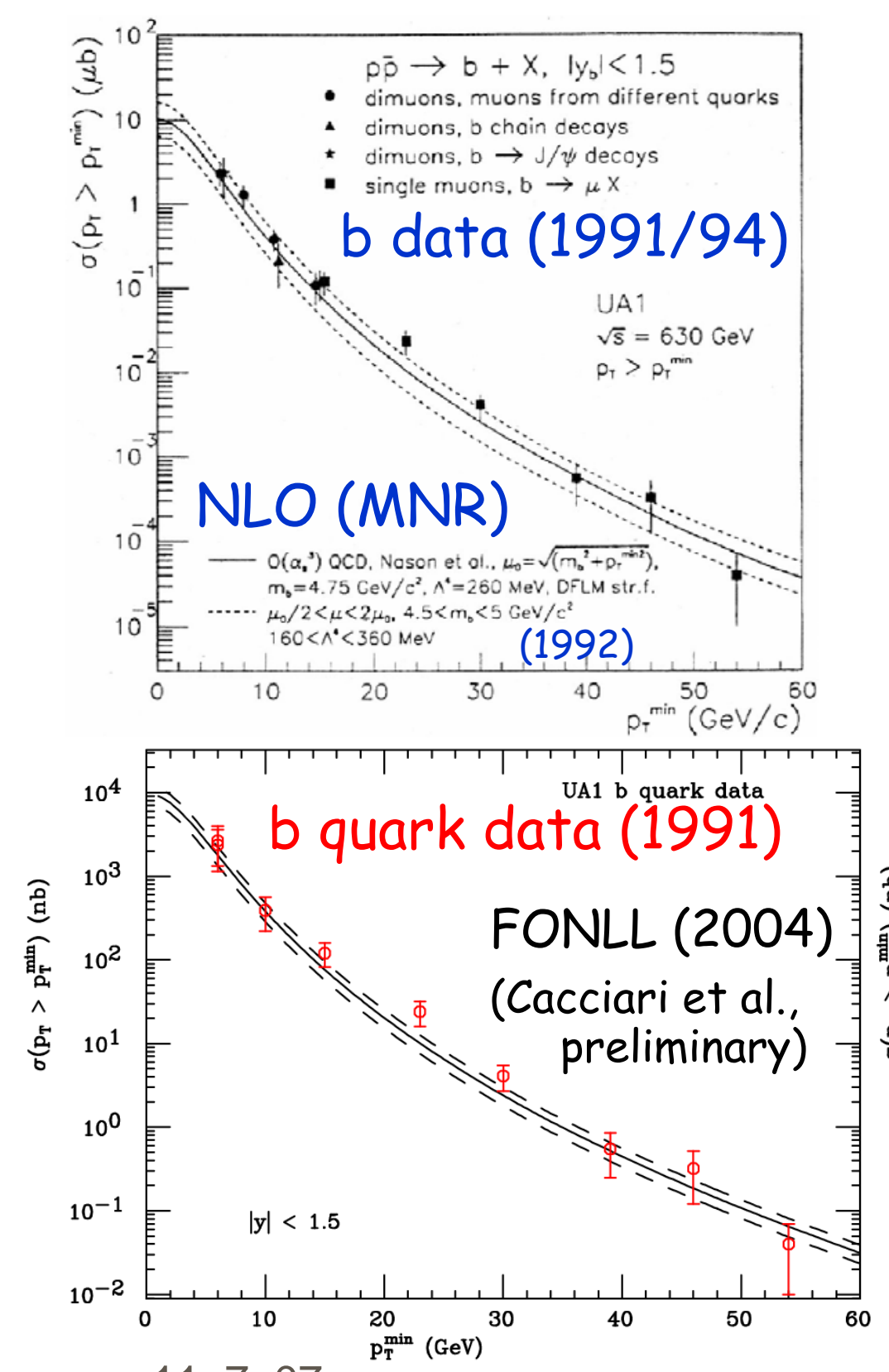

11. 7.07
NLO (1992) and FONLL (2004) agree with each other and with data (1991/94)

(fragm. and decay spectra, br. ratios, ... were all consistently tuned in $M C$ to measured data)

FONLL (2004) agrees with data (1991) $a+b$ quark, $B$ hadron, and muon level

UA1, PLB 256 (1991) 112 UA1, Z.Phys.C 61 (1994) 41 MNR, Nucl.Phys.B 373 (1992) 295 Cacciari et al., JHEP 0407 (2004) 33

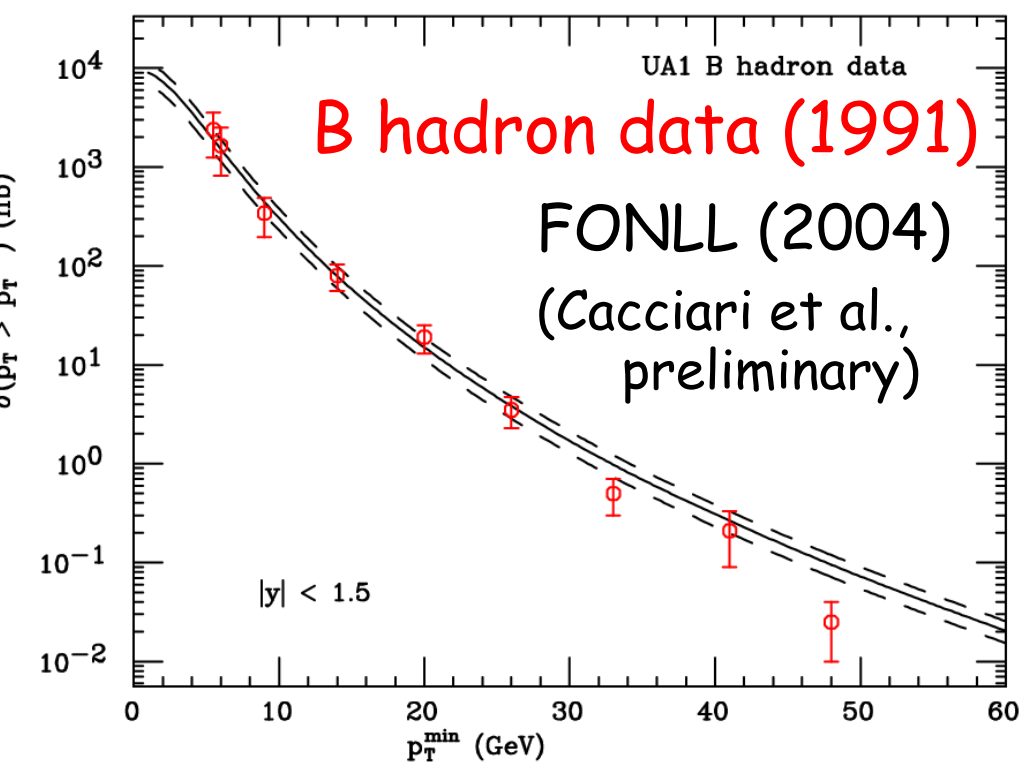

Photon07, Paris

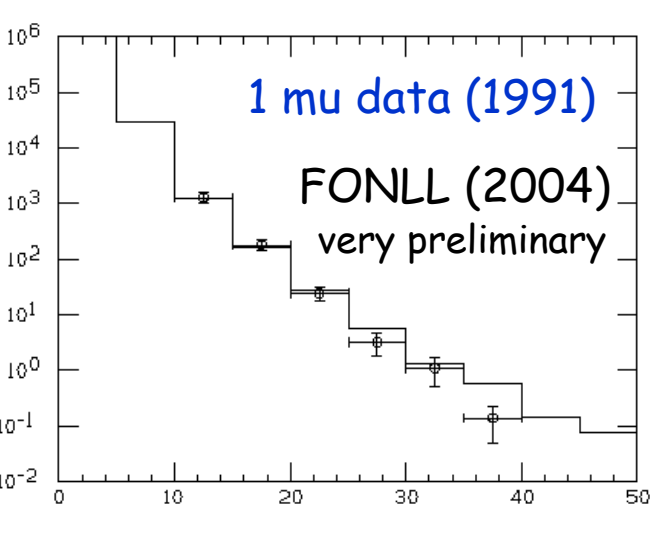




\section{Charm in photoproduction at HERA}

ZEUS
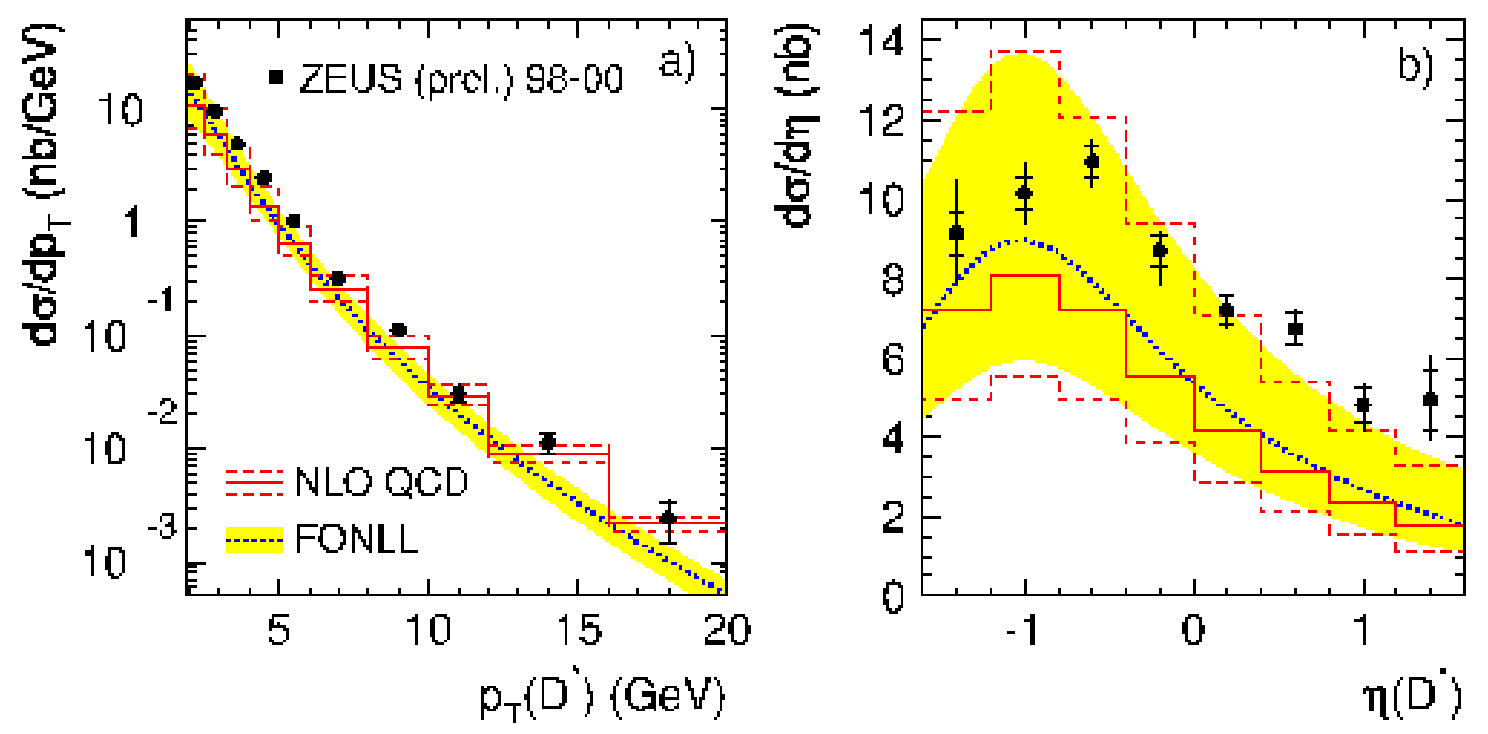

$\Rightarrow$ Do not expect major change for $b$ at HERA from

NLO (no resummation)

$\rightarrow$ FONLL (with resummation)

but would be nice to have
QCD calculations using CTEQ5M1 + AFG structure functions $m_{c}=1.5+-0.2 \mathrm{GeV}, \quad \mu_{0}{ }^{2}=m_{c}{ }^{2}+p_{T}{ }^{2}$, $\mu_{r}=\mu_{f}=\mu, \quad \mu_{0} / 2<\mu<2 \mu_{0}$ $f\left(c->D^{*}\right)=0.235$ $\mathrm{e}_{\text {Peterson }}=0.035$ (FO NLO), 0.02 (FONLL)

NLO (FMNR) reasonable agreement some differences at forward $\eta$

FONNL (Cacciari et al.) similar, not better at large $p_{T}$ 